OPEN ACCESS

Edited by:

Haiwei LuO,

The Chinese University of Hong Kong,

Hong Kong

Reviewed by:

Paris Sepulveda Lavin,

University of Antofagasta, Chile

Sergio Leiva,

Universidad Austral de Chile, Chile

*Correspondence:

Runying Zeng

zeng@tio.org.cn;

zengrunying@126.com

${ }^{\dagger}$ These authors have contributed equally to this work

Specialty section:

This article was submitted to Evolutionary and Genomic

Microbiology,

a section of the journal

Frontiers in Microbiology

Received: 20 March 2018

Accepted: 25 July 2018

Published: 14 August 2018

Citation:

Qu W, Lin D, Zhang Z, Di W, Gao B and Zeng $R$ (2018) Metagenomics

Investigation of Agarlytic Genes and Genomes in Mangrove

Sediments in China: A Potential Repertory for Carbohydrate-Active Enzymes. Front. Microbiol. 9:1864. doi: 10.3389/fmicb.2018.01864

\section{Metagenomics Investigation of Agarlytic Genes and Genomes in Mangrove Sediments in China: A Potential Repertory for Carbohydrate-Active Enzymes}

\author{
Wu Qu't, Dan Lin't, Zhouhao Zhang'2, Wenjie Diं3, Boliang Gao' and Runying Zeng ${ }^{3,4 *}$ \\ 'School of Life Sciences, Xiamen University, Xiamen, China, ${ }^{2}$ Novogene Bioinformatics Technology Co., Ltd., Tianjin, China, \\ ${ }^{3}$ Key Laboratory of Marine Genetic Resources, Third Institute of Oceanography, State Oceanic Administration, Xiamen, \\ China, ${ }^{4}$ Key Laboratory of Marine Genetic Resources, Xiamen, China
}

Monosaccharides and oligosaccharides produced by agarose degradation exhibit potential in the fields of bioenergy, medicine, and cosmetics. Mangrove sediments (MGSs) provide a special environment to enrich enzymes for agarose degradation. However, representative investigations of the agarlytic genes in MGSs have been rarely reported. In this study, agarlytic genes in MGSs were researched in detail from the aspects of diversity, abundance, activity, and location through deep metagenomics sequencing. Functional genes in MGSs were usually incomplete but were shown as results, which could cause virtually high number of results in previous studies because multiple fragmented sequences could originate from the same genes. In our work, only complete and nonredundant (CNR) genes were analyzed to avoid virtually high amount of the results. The number of CNR agarlytic genes in our datasets was significantly higher than that in the datasets of previous studies. Twenty-one recombinant agarases with agarose-degrading activity were detected using heterologous expression based on numerous complete open-reading frames, which are rarely obtained in metagenomics sequencing of samples with complex microbial communities, such as MGSs. Aga2, which had the highest crude enzyme activity among the 21 recombinant agarases, was further purified and subjected to enzymatic characterization. With its high agarosedegrading activity, resistance to temperature changes and chemical agents, Aga2 could be a suitable option for industrial production. The agarase ratio with signal peptides to that without signal peptides in our MGS datasets was lower than that of other reported agarases. Six draft genomes, namely, Clusters 1-6, were recovered from the datasets. The taxonomic annotation of these genomes revealed that Clusters 1, 3, 5, and 6 were annotated as Desulfuromonas sp., Treponema sp., Ignavibacteriales spp., and Polyangiaceae spp., respectively. Meanwhile, Clusters 2 and 4 were potential new species. All these genomes were first reported and found to have abilities of degrading various important polysaccharides. The metabolic pathway of agarose in Cluster 4 was also speculated. Our results showed the capacity and activity of agarases in the MGS microbiome, and MGSs exert potential as a repertory for mining not only agarlytic genes but also almost all genes of the carbohydrate-active enzyme family.

\footnotetext{
Keywords: mangrove sediments, metagenomics, deep-sequencing, CAZyme, agarlytic gene
} 


\section{INTRODUCTION}

Given the demand for bioenergy, researchers have isolated numerous genes and genomes with biomass-degrading functions (Harris et al., 2010; Langston et al., 2011; Sheydina et al., 2014; Yang et al., 2016) to produce fermentable monosaccharides. To date, most of these works have focused on screening cellulase genes, and cellulose is the usual material for producing fermentable monosaccharides. However, cellulose application is limited by two reasons. First, cellulose is extremely difficult to dissolve in water. Therefore, cellulases should be adsorbed on the cellulose surface to be hydrolyzed and thus have low catalytic efficiency (Lynd et al., 2002; Zhang and Lynd, 2003). Second, cellulose has high molecular weight (MW) and exists in natural plants; lignin is tightly intertwined with cellulose and has also high MW. Therefore, natural cellulose fibrils not only exclude water but also cellulases (Lynd et al., 2002; Zhang and Lynd, 2003). Therefore, pretreatment using molten salts (i.e., $\mathrm{LiCl}_{3} \mathrm{H}_{2} \mathrm{O}$ ), nonaqueous solvent systems (i.e., $\mathrm{N}, \mathrm{N}$-dimethylacetamide/LiCl, $\mathrm{DMSO} / \mathrm{SO}_{2}$, and $N$-methylmorpholine- $N$-oxide) (Heinze and Liebert, 2001), supercritical carbon dioxide (SC- $\mathrm{CO}_{2}$ ) (Kim and Hong, 2001), and ionic liquids (Kuo and Lee, 2009) is needed to separate lignin from cellulose and increase cellulose solubility. According to a previous study, the requirement for pretreatment restricts the application of cellulose hydrolysis (Jeoh et al., 2007). Meanwhile, the cost of above-mentioned cellulose pre-treatment is very expensive. Therefore, producing fermentable monosaccharides through cellulose hydrolysis by cellulases still has limited application. Agarose extracted from red algae has attracted increasing attention in recent years because of its high yield similar to cellulose (Roesijadi et al., 2010; Wargacki et al., 2012; Yanagisawa et al., 2013). Agarose exhibits properties that address the limitations of cellulose. Individual molecular agarose shows no complex coexistence with other molecular particles. Moreover, the solubility of agarose is higher than that of celluloses, and agarases could directly function on agarose dissolved in water. Accordingly, the enzymatic hydrolysis efficiency of agarose is significantly increased compared with that of celluloses. Therefore, agarose can be simply extracted and dissolved by heating, which is easier and cost saving than the current pre-treatment methods for cellulose. Hence, agarose is a potential alternative to cellulose for fermentable monosaccharide production. Ethanol and hydrogen $\left(\mathrm{H}_{2}\right)(\mathrm{Kim}$ et al., 2012; Wu et al., 2017) are fermentation products obtained after agarose saccharification using three types of agarlytic enzymes, including $\alpha$-agarase, $\beta$-agarase, and $\alpha-1,3$ neoagarobiose hydrolase (NABH) (Suzuki et al., 2003; Ohta et al., 2005; Watanabe et al., 2017). Neoagarooligosaccharides (NAs) produced by $\beta$-agarase degradation also exert many biological activities (Hong et al., 2017a,b), including anti-oxidation, antiinflammation (Wang et al., 2004; Enoki et al., 2010), probiotic, and whitening properties (Hu et al., 2006). Therefore, the demand for enzymes with outstanding agarose-degrading activity is high.

Appropriate environmental samples containing considerable amount of these genes are required to isolate agarlytic genes. For example, cattle rumen and termite intestines have been reported as excellent sources of cellulase genes (Warnecke et al., 2007; Hess et al., 2011; Liu et al., 2011). However, a repertory of the agarlytic genes has never been proved to date. Sediments of mangrove ecosystems have extremely complex microbial communities (Bharathkumar et al., 2008; Dias et al., 2009; Jiang et al., 2013; Wu et al., 2016). Leaves, crustacean carcasses, and algal debris are abundant in mangrove sediments (MGSs), which are potential sources of agarlytic genes. A few agarase genes have been isolated from MGSs by using pure culture and metagenomics library methods (Shome et al., 2000; Mai et al., 2016). However, these methods are biased and time consuming in making a general survey of the capacity of agarlytic genes in MGSs. High-throughput sequencing provides a fresh perspective to investigate sequences in environmental samples integrally and without bias through simple operations. This method can detect genes, determine the abundance and diversity of certain genes (Anantharaman et al., 2016; Slaby et al., 2017), and draft genomics information in environmental samples (Leung et al., 2011; Girotto et al., 2016). However, only few studies have investigated genes in MGSs (Andreote et al., 2012; Thompson et al., 2013; Alzubaidy et al., 2016) by metagenomics sequencing because of the extremely complex microbial community. Only one study reported on CAZyme genes in MGSs (Thompson et al., 2013). Furthermore, these reports have some drawbacks as follows. (i) The genes obtained in these datasets are usually incomplete, but all the openreading frames (ORFs) are shown as the results, regardless of completeness. Therefore, the true number of genes in these studies will be lower than those in the results because the datasets may contain nonoverlapping fragments from the same ORF. (ii) Furthermore, the massive incomplete genes complicated the application of heterologous expressions to verify the catalytic activities of the corresponding proteins. (iii) Lastly, the draft genomes are important to understand the metabolic pathways of agarose in nonculturable microorganisms, which make up the majority of the microbial community in nature (Zengler et al., 2005; Lengeler et al., 2009; D’Onofrio et al., 2010) and cannot be assembled from these datasets because of the low coverage. From these studies, the actual capacity, diversity, and activity of the agarlytic genes in MGSs remain unexplored and unconfirmed.

To address the above mentioned concerns and conduct a comprehensive study on the agarlytic genes and related genomes in MGSs, we deeply sequenced the environmental DNA (eDNA) of MGSs in China by using the Illumina platform. We obtained $\sim 300 \mathrm{~Gb}$ of data in total, which is the maximum data size among the metagenomics projects of MGSs. The amount, diversity, and completeness of the agarlytic genes were significantly increased compared with the previous works. Moreover, we focused on the CNR agarlytic genes. Most of these CNR agarlytic genes were unreported in the NCBI nr database. Twenty-one recombinant agarases with agarose-degrading activity were discovered using heterologous expression in Escherichia coli cells. Aga2 showed potential for practical applications due to its high activity and stability against thermo and many chemical agents. The signal peptide analysis showed that the agarase ratio of with signal peptides to that without signal peptides in our MGS datasets was obviously lower than that of the agarases reported in NCBI nr 
database. Moreover, we assembled six draft genomes from our datasets with unsupervised machine learning. We also analyzed the abilities for polysaccharide degradation in our study. The results proved that MGSs are potential repertories of agarlytic genes and almost all families of CAZyme genes.

\section{MATERIALS AND METHODS}

\section{Sampling and Physicochemical Analysis}

Sediment samples were collected from a mangrove forest located in Longhai City, Fujian, China (Zini Mangrove Nature Reserve; $\left.24^{\circ} 20^{\prime} \mathrm{N}, 117^{\circ} 45^{\prime} \mathrm{E}\right)$. To increase the abundance of the agarase genes, we buried 1\% agar with the sediments in five sites of the middle of mangrove with $2-\mathrm{m}$ interval for 20 days. Sediments from the other three sites without any treatment served as control groups were also collected. Approximately $500 \mathrm{~g}$ each of the agar-enriched (enrichment groups) and unenriched sediments (control groups) was collected in several $50 \mathrm{~mL}$ sterile centrifugal tubes. The sediment samples were transported to the laboratory and stored in an ultra-low temperature freezer at $-80^{\circ} \mathrm{C}$.

Sediment temperature was measured with an alcohol thermometer in situ. Sediments salinity was determined by using a handheld salinity meter (ATAGO, Japan). The humidity of sediments was defined as the percentage of the weights of dry and wet samples and was measured accordingly. $\mathrm{pH}$, total nitrogen, total phosphorus, and total carbon were measured by Qingdao Hengli Testing Co., Ltd. (China) in accordance with the national standards of China.

\section{eDNA Extraction}

The Powersoil DNA Isolation Kit (MO BIO, United States) was used to extract the eDNA of MGSs in accordance with the manufacturer's instructions except that the DNA was eluted by sterile water instead of Solution C6 provided in the kit. Approximately $0.5 \mathrm{~g}$ of each sediment sample was used in the DNA extraction. Three replications of extraction and purification were performed and pooled together to avoid extraction bias. The DNA purity and integrity were determined by $1 \%$ agarose electrophoresis and NanoDrop 2000 (Thermo Scientific, United States).

\section{Sequencing of 16S rRNA Genes}

Primer pairs 338F and 806R with barcodes (Fan et al., 2014) were chosen to amplify the $16 \mathrm{~S}$ rRNA genes from MGSs. According to former studies (Xiao et al., 2015; Zhang et al., 2015; Zheng et al., 2015), the polymerase chain reaction (PCR) system and programs are listed in Supplementary Tables S1, S2, respectively.

Polymerase chain reaction products were used to construct a paired-end library of Illumina Miseq (Illumina Inc., United States). The low-quality bases $(<20)$ were removed. The paired reads were assembled based on the overlapping sequences. The identification and removal of chimeras, operational taxonomic units (OTUs) clustering, and taxonomic assignment were performed using Qiime (Caporaso et al., 2010). The beta-diversity were analyzed using the $\mathrm{R}$ software ( $\mathrm{R}$ Core Team, 2016).

\section{Metagenomics Sequencing}

Environmental DNA was used to construct the $400 \mathrm{bp}$ and $6 \mathrm{~kb}$ library, which were sequenced using an Illumina HiSeq (Illumina Inc., United States) by Novogene Technology Co., Ltd. (China). Approximately $300 \mathrm{~Gb}$ of data was generated. The reads with more than 40 nt low-quality bases (quality value $\leq 38$ ) were removed. Meanwhile, the reads with more than $10 \mathrm{nt}$ " $\mathrm{N}$ " bases were filtered out of the datasets. Lastly, the reads overlapping more than $15 \mathrm{nt}$ bases with the adapters were also removed. Accordingly, the clean data were obtained. All clean reads were assembled using MEGAHIT (Li et al., 2015) with the parameter of "-presets meta-large," and the scaffolds were broken at $\mathrm{N}$ into the scaftigs (continuous sequences within scaffolds) (Mende et al., 2012; Nielsen et al., 2014). The scaftigs with the length of $\geq 500 \mathrm{nt}$ were used for further analysis (Li et al., 2014; Sunagawa et al., 2015). The ORFs in the scaftigs ( $\geq 500 \mathrm{bp}$ ) were predicted by MetaGeneMark. A nonredundant gene catalog was obtained after processing by using the CD-HIT software. Gene abundance was calculated based on the number of reads mapped to the genes and the length of the genes. The databases, including the CAZy Database ${ }^{1}$, Kyoto Encyclopedia of Genes and Genomes $\left(\mathrm{KEGG}^{2}\right)$, and Evolutionary Genealogy of Genes: Nonsupervised Orthologous Groups ${ }^{3}$, were used for the functional annotation of genes. Unigenes were aligned with the sequences in these databases by using the DIAMOND software (blastp, cut-off $E$-value of $1 e-5)$, and the best hits were chosen as the functional annotations. The amino acid sequences of the proteins annotated as agarlytic genes were realigned in the NCBI nr database by using the BLAST software (version 2.2.26) to determine the novelty of these sequences.

To examine the sequencing accuracy, we randomly picked and amplified 100 sequences from our datasets (Supplementary Table S3). The sequences of the PCR products were determined by Shanghai Majorbio Bio-Pharm Technology Co. (China) by using an ABI 3730xl DNA Analyzer (Thermo Scientific, United States). The results were compared with the original sequences through DNAman software.

\section{Prediction of the Optimum Temperature and Signal Peptide}

The optimum temperatures of the agarlytic genes with complete ORFs were predicted using the method described in a previous report (Chu et al., 2016). Meanwhile, the signal peptides were predicted using the SignalP script (SignalP 4.1 for Linux) (Petersen et al., 2011). All the complete agarase genes in the enrichment and control groups were selected for the signal peptide prediction. For further study, 424 agarases (Supplementary File), which were found in the culturable microorganisms, metagenomic libraries, and genome sequencing, were selected for signal peptide prediction by using the same script.

\footnotetext{
${ }^{1}$ http://www.cazy.org/

${ }^{2}$ http://www.kegg.jp/

${ }^{3}$ http://eggnog.embl.de/
} 


\section{Chimerism Correction}

The contig coverage was calculated using the bedtools based on the read and insert mapping performed by Bowtie. Coverage difference (CD) was calculated as follows: $\mathrm{CD}=\operatorname{abs}(\mathrm{m} 1-\mathrm{m} 2) / \min (\mathrm{m} 1, \mathrm{~m} 2)$, where $\mathrm{m} 1$ and $\mathrm{m} 2$ are the coverage of the two adjacent windows. Contig would be broken at where $\mathrm{CD}$ was over 0.75 regardless it was based on read or insert mapping. After the correction, all datasets were pooled together, and deredundancy was performed for further genome assembly.

\section{Genome Assembly}

CONCOCT (Alneberg et al., 2014) was employed to bin the contigs $(>2 \mathrm{~kb})$ into clusters on the basis of the sequence composition and coverage. The gene integrity and heterozygosity of each cluster were evaluated by Prodigal (Hyatt et al., 2010), and the alignment was conducted using RPS-BLAST ( $E$-value: $1.0 e-3$; coverage: $50 \%$ ) in the NCBI-COG database. On the basis of the reference, the clusters with coverage of single-copy genes above $75 \%$ were selected for further genome assembly. The reads from the $400 \mathrm{bp}$ and $6 \mathrm{~kb}$ libraries that were mapped to the selected clusters were isolated with SOAP (version 2.21) (Li et al., 2008). Genome assembly was performed using SPAdes (version 3.10) (Bankevich et al., 2012) with different $K$-mer values (17, 27, $37,47,57,67$, and 77). The DNA fragments shorter than $500 \mathrm{bp}$ were removed. Six draft genomes were obtained for further study through these methods.

For the taxonomic annotation of these draft genomes, BLASTn was first used to align the genomic sequences to NCBI nr database with the parameters of "-a 4 -e 1e-5 -b 50." Sequences with $E$-value $\geqq 10 \times$ minimum $E$-value were picked up (Qin et al., 2010). To further study the taxonomy of these sequences, least common ancestors (LCAs) method was performed using MEGAN software (Huson et al., 2011), and the taxonomy of the sequences was confirmed based on the taxonomic result before the first branch. Subsequently, the taxonomic annotation results of the six draft genomes were obtained according to the percentage of annotated results of the sequences within each draft genome.

\section{Agarase Gene Expression}

Thirty agarase genes (aga-aga30) with random selection were expressed in E. coli. The primers designed based on ORFs are listed in Supplementary Table S4. All PCR products were ligated into the pEASY-Blunt E2 Expression Vector (TransGen Biotech, China) and transformed into E. coli BL21 (DE3) cells. The recombinant proteins were induced by $0.05 \mathrm{mM}$ isopropyl $\beta$-D-1-thiogalactopyranoside at $16^{\circ} \mathrm{C}$ for $24 \mathrm{~h}$.

\section{Agarase Activity Assay}

Crude agarase was produced after ultrasonicating the host cells. The protein concentration was measured using a Pierce BCA Protein Assay Kit (Thermo Scientific, United States), following the manufacturer's instructions. Agarase activity $(\mathrm{U})$ was defined as the amount of enzymes that produced $1 \mu \mathrm{M}$ of reducing sugar per minute under the assay conditions. The concentration of reducing sugar was measured using the 3,5-dinitrosalicylic acid (DNS) method (Miller, 1959) by using D-galatose as a standard. Different buffer solutions (acetate- $\mathrm{NaOH}, \mathrm{pH} 3.0-6.0$; phosphate- $\mathrm{NaOH}, \mathrm{pH} 7.0-8.0$; Tris- $\mathrm{HCl}$, and $\mathrm{pH} 9.0-10.0$ ) and temperatures $\left(20-80^{\circ} \mathrm{C}\right)$ were used to determine the effects of $\mathrm{pH}$ and temperature on the agarase activity. The reaction mixture was composed of $5 \mu \mathrm{L}$ of crude agarose and $245 \mu \mathrm{L}$ of reaction buffer solution with $0.2 \%$ dissolved agarose. After incubating at a certain temperature $\left(20-80^{\circ} \mathrm{C}\right)$ for $30 \mathrm{~min}, 750 \mu \mathrm{L}$ of DNS was added and heated at $100^{\circ} \mathrm{C}$ for $10 \mathrm{~min}$. The absorbance was measured at a wavelength of $550 \mathrm{~nm}$. The heat-inactivated crude agarase served as the negative control. Each experiment was performed in triplicate.

\section{Purification and Activity Staining of Recombinant Agarase}

Aga2 with the crude highest agarose-degrading activity was further studied after purification with HisPur Ni-NTA spin columns (Thermo, United States). Purified proteins were eluted by using $200 \mathrm{mM}$ imidazole solution. Furthermore, purified proteins were dialyzed against $20 \mathrm{mM}$ PBS (pH 7.4) to replace midazole and stored at $-20^{\circ} \mathrm{C}$ with $30 \%$ glycerol (final concentration). The protein concentration was detected using Pierce BCA Protein Assay Kit (Thermo Fisher Scientific, United States) by using bovine serum protein as a standard. All the experiments followed the manufacturer's instructions. The purified Aga2 was analyzed by $10 \%$ sodium dodecyl sulfatepolyacrylamide gel electrophoresis (SDS-PAGE) and stained with Coomassie Brilliant blue.

After SDS-PAGE, activity staining of Aga2 was conducted. SDS in the gel was removed by rinsing the gel in PBS ( $\mathrm{pH}$ 7.4) for three times at about 30 min each time. Then, the gel was placed on $1 \%$ agar plate, and the plate was incubated at $30^{\circ} \mathrm{C}$ for $12 \mathrm{~h}$. Iodine solution was used for staining the agar plate to show a clean zone at the Aga2 position.

\section{Determination of Thermal and $\mathrm{pH}$ Stability of Aga2}

To determine the Aga2 stability against the temperature, the enzyme solution was preincubated at $20-80^{\circ} \mathrm{C}$ for $0.5 \mathrm{~h}$ at $\mathrm{pH}$ 7.0. In addition, the enzyme solution was preincubated at $50^{\circ} \mathrm{C}$ in $\mathrm{pH} 4.0-9.0$ for $0.5 \mathrm{~h}$ to determine the stability of Aga2 against the $\mathrm{pH}$. After preincubation, the residual activity of the enzyme solution was measured as mentioned above, and the activity in the absence of any treatment was defined as $100 \%$. All experiments were performed in triplicates.

\section{Effects of Reagents on Aga2 Activity}

The effects of metal ions and chelating agent were measured by determining the Aga2 activity with $\mathrm{Ca}^{2+}, \mathrm{Ni}^{2+}, \mathrm{Fe}^{3+}$, $\mathrm{Zn}^{2+}, \mathrm{Ag}^{+}, \mathrm{Mn}^{2+}, \mathrm{Cu}^{2+}$, and $\mathrm{Al}^{3+}$ at a final concentration of $1 \mathrm{mmol} / \mathrm{L}$. The effect of denaturants was determined by mixing the reaction mixture with different concentrations of SDS, dithiothreitol (DTT), and beta-mercaptoethanol (beta-Me). The effects of salt concentration and metal chelator were measured with 10 and $100 \mathrm{mM} \mathrm{NaCl}$ and EDTA-2Na (pH 8.0), respectively. 
Agarase activity was measured. The Aga2 activity in the absence of any treatment was $100 \%$. All experiments were performed in triplicates.

\section{Determination of Agarase Activity in MGSs}

The extracellular agarase activity in the sediments was determined as follows. Initially, $10 \mathrm{~g}$ of fresh sediments and $10 \mathrm{~mL}$ of $2 \%$ agarose dissolved in phosphate-buffered saline (PBS) (10 mM, pH 7.4) were diluted to $100 \mathrm{~mL}$ with PBS. The mixture was incubated at $35^{\circ} \mathrm{C}$ for $72 \mathrm{~h}$. The reaction mixture $(2 \mathrm{~mL})$ was added with $6 \mathrm{~mL}$ of DNS and heated at $100^{\circ} \mathrm{C}$ for $10 \mathrm{~min}$. Absorbance was recorded at a wavelength of $550 \mathrm{~nm}$.

Total agarase activity was determined as follows. A total of $10 \mathrm{~g}$ of fresh sediments were adequately suspended in $50 \mathrm{~mL}$ of PBS. The intracellular agarases were released after ultrasonication. The lysate and $10 \mathrm{~mL}$ of $2 \%$ agarose were diluted to $100 \mathrm{~mL}$ with $\mathrm{PBS}$, and the remaining processes were performed as described above. The intracellular activity was obtained by subtracting the extracellular activity from the total activity. Sterilized sediments served as the negative controls. The agarase activity was defined as the mass $(\mathrm{mg})$ of reducing sugar produced by per gram of sediment after incubation for $72 \mathrm{~h}$.

\section{Nucleotide Sequence Accession Numbers}

The Illumina HiSeq sequence data from this study were submitted to the EMBL-EBI European Nucleotide Archive under the Accession Nos. ERR2179512 (Mgv-CK), ERR2179511 (Mgv-B-L6000), and ERR2179510 (Mgv-B). The nucleotide sequences of the 21 agarase genes that were conformed with agar-degrading activity were submitted to GenBank under the Accession Nos. MG280837 (aga1), MG383539 (aga2), MG383540 (aga4), MG383541 (aga5), MG383542 (aga6), MG383543 (aga7), MG383544 (aga9), MG383545 (aga10), MG383546 (aga12), MG383547 (aga13), MG383548 (aga14), MG383549 (aga15), MG383550 (aga16), MG383551 (aga18), MG383552 (aga19), MG383553 (aga21), MG383554 (aga22), MG383555 (aga23), MG383556 (aga27), MG383557 (aga28), and MG383558 (aga29).

\section{RESULTS}

\section{Sample Description}

For all the enrichment groups, the agar in the sediments changed dramatically, showing liquefaction and numerous holes. This phenomenon suggested agar degradation. However, the enrichment groups were changed into two colors, namely, yellow and black, respectively. In detail, three sites were changed into black and two sites were changed into yellow. Physicochemical parameters of the enrichment groups were measured along with the control groups (without any treatment). Results showed that there was no obvious difference among the samples from enrichment groups (Table 1) except the colors. Therefore, the enrichment groups were classified into two groups according to the color, namely, Mgv-Y (yellow) and Mgv-B (black). Meanwhile, the control groups were named Mgv-CK. The eDNA from same group was pooled together for further analyses.

\section{Change in the Microbial Diversity in MGSs After Enrichment}

We analyzed the diversity of the 16S rRNA genes to confirm whether the agar enrichment was effective and to identify which of the two enrichment samples (Mgv-Y and Mgv-B) was more effectively agar-enriched. The sequencing depth was sufficient according to rarefaction curve (Supplementary Figure S1).

The alpha-diversity of microbes in MGSs varied after enrichment (Table 2). The abundance represented by abundancebased coverage estimator (ACE) and chao indices increased in Mgv-Y and Mgv-B groups; however, the microbial diversities represented by Shannon and Simpson indices of Mgv-Y and Mgv-B groups were lower than Mgv-CK, indicating that agar enrichment could increase the microbial abundance but reduce the diversity in the sediments. Moreover, compared with Mgv$\mathrm{Y}$, the variation of microbial alpha-diversity was more obvious in Mgv-B.

The beta-diversity results showed that the community of the two enrichment groups was significantly changed relative to that of Mgv-CK (Figure 1). Proteobacteria was the dominant phylum in MGSs, followed by Bacteroidetes, Chloroflexi, Acidobacteria, and Firmicutes. After enrichment, the abundance of Bacteroidetes and Firmicutes increased in Mgv-B and Mgv-Y, and most obvious increasing occurred in Mgv-B (Figure 1A). The microbial community in Mgv-Y was still similar to the control group (Mgv-CK). However, the microbial Mgv-B structure was significantly shifted and phylogenetically distanced from the others (Figure 1B). On the genus level, several genera, such as Acidaminococcus, Aeromonas, Arcobacter, Bacteroides, and Ruminococcus were enriched in Mgv-B and Mgv-Y. Among these genera, 10/16 was reported with the ability of glycoside hydrolysis (Figure 1C and Supplementary Table S5). Similarly, the most significant change occurred in Mgv-B (Figure 1C and Supplementary Table S5).

Therefore, eDNA of Mgv-B with the most obvious changes after enrichment was further sequenced along with the control group (Mgv-CK).

\section{Metagenomics Data Overview}

In total, $\sim 300 \mathrm{~Gb}$ of the data was generated from the enrichment and control groups (Supplementary Table S6). Moreover, 25,172,487 ORFs (13,049,096 from the control group and $12,123,391$ from the enrichment group) were found in our datasets. Among these sequences, nearly 1/5-1/4 had complete ORFs (Supplementary Table S7). To examine the accuracy of sequencing and assembly, we randomly sequenced and amplified 100 sequences by using PCR, and 97 out of the 100 amplified and sequenced fragments were found with more than $97 \%$ identity of the original sequences. This result suggested the high accuracy of our datasets (Supplementary Figure S2).

Except for the microbial community, the number of the function genes was varied (Supplementary Figure S3). The gene 
abundance related to "Environmental Information Processing," "Genetic Information Processing," and "Cellular Processes" increased after enrichment. For example, the abundance of ABC transporter genes increased from $1.1 \%$ to $1.5 \%$. The abundance of ribosome genes increased from $0.5 \%$ to $0.9 \%$. The gene abundance of "Cellular Processes" evidently increased. Moreover, after agar enrichment, the abundance of bacterial chemotaxis and flagellar assembly genes increased from $0.1 \%$ to $0.4 \%$ and from $0.08 \%$ to $0.3 \%$, respectively.

\section{CAZyme Genes in MGSs}

To investigate the capacity of CAZymes in MGSs, the CNR CAZyme genes of the control group were emphatically analyzed. These results included the auxiliary activities, carbohydratebinding modules (CBMs), carbohydrate esterases, glycoside hydrolases (GHs), glycosyl transferases, and polysaccharide lyases (PLs) (Supplementary Figure S4 and Supplementary Table S8).

In the Mgv-CK datasets (control group), 12,195 CNR GH genes were found (Supplementary Table S9). This value was significantly higher than the number in the previous mangrove studies. GH 3 and GH 13, which are known as the important families related to polysaccharide hydrolysis, were abundant in the MGS datasets. In addition to GH 3 and 13, other families associated with cellulose hydrolysis, such as $\mathrm{GH} 6, \mathrm{GH} 12, \mathrm{GH}$ 17, CBM 2, and CBM 3, were also detected with high abundance in the datasets. The lysozyme family GH 23 was as well abundant. The pectin lyase families, namely, PL 1 and PL 9, achieved the highest numbers in the PL family.

The number of the CNR genes from each GH family from our datasets was compared with those from other datasets, including

TABLE 1 | Physicochemical parameters of the sediment samples*

\begin{tabular}{|c|c|c|c|c|c|c|c|c|}
\hline Group & Sample No. & $\mathrm{pH}$ & Temperature $\left({ }^{\circ} \mathrm{C}\right)$ & Salinity $(\% 0)$ & Humidity (\%) & $\mathrm{TC}(\mathrm{g} / \mathrm{kg})$ & $\mathrm{TP}(\mathrm{g} / \mathrm{kg})$ & $\mathrm{TN}(\mathrm{g} / \mathrm{kg})$ \\
\hline \multirow[t]{3}{*}{ Mgv-B } & 1 & 6.41 & 25.4 & 15.1 & 20.9 & 5.9 & 0.127 & 0.325 \\
\hline & 2 & 6.33 & 24.5 & 16.0 & 16.8 & 5.3 & 0.109 & 0.344 \\
\hline & 3 & 6.94 & 25.5 & 15.4 & 15.2 & 5.1 & 0.174 & 0.239 \\
\hline \multirow[t]{2}{*}{ Mgv-Y } & 4 & 6.24 & 26.2 & 15.0 & 23.5 & 4.9 & 0.146 & 0.368 \\
\hline & 5 & 6.78 & 24.9 & 15.3 & 21.1 & 5.4 & 0.109 & 0.308 \\
\hline \multirow[t]{3}{*}{ Mgv-CK } & 6 & 6.51 & 26.7 & 16.7 & 18.4 & 3.8 & 0.140 & 0.408 \\
\hline & 7 & 6.15 & 25.8 & 16.4 & 23.1 & 4.2 & 0.135 & 0.586 \\
\hline & 8 & 6.43 & 25.1 & 15.6 & 22.9 & 4.6 & 0.156 & 0.301 \\
\hline
\end{tabular}

*TC, TP, and TN represented total carbon, total phosphorus, and total nitrogen, respectively.

A

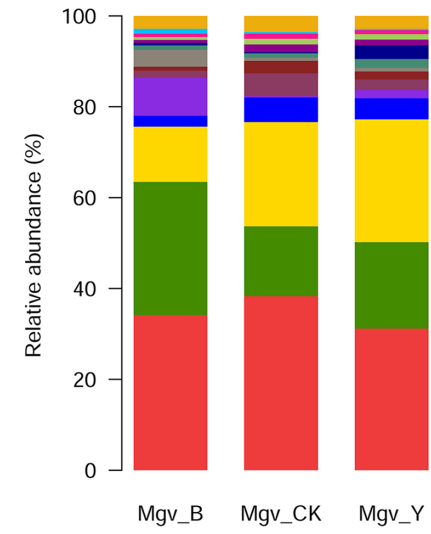

B

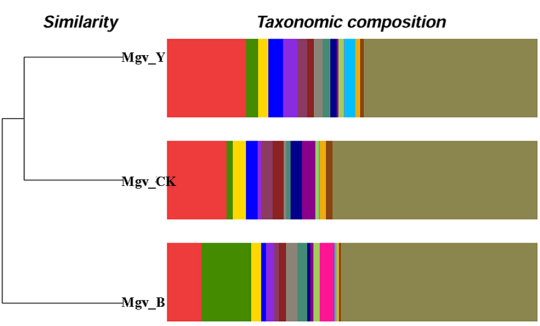

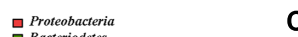

Bacteriodetes - Chloroflexi
- Acidobacteria
Firmicutes - Acidobact
- Fitrospites - Chlorobi
- Spirochaetae
Planctomycat - Splanctomyctae
Candidate_division_TM7 - Bacteria_unclassified
Gemmatimonaderes
Candidate_division_ws ㅁ Candidate-dia
ㅁ Fusobacteri
Others

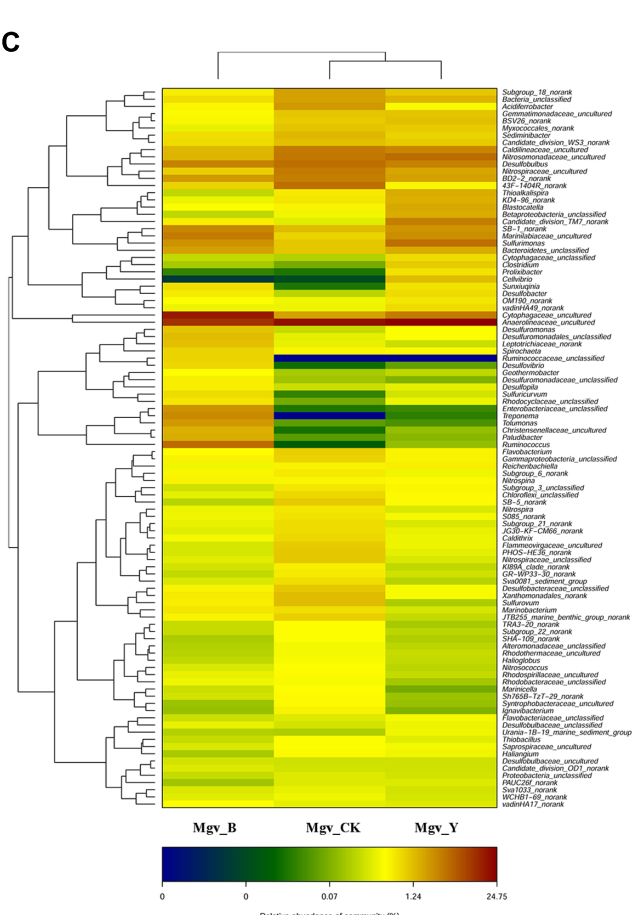

FIGURE 1 | Diversity of 16 S rRNA genes in the enrichment and control groups. (A) Community composition (phylum level). (B) Sample clustering (genus level). (C) Heatmap of community composition (genus level). 
TABLE 2 | Alpha-diversity indices of microbial 16S rRNA genes in the sediment samples after subsampling.

\begin{tabular}{lccccc}
\hline Group & Reads No. & ACE & Chao & Shannon & Simpson \\
\hline Mgv-B & 59755 & 1991 & 2025 & 5.94 & 0.0118 \\
Mgv-Y & 59755 & 1962 & 1986 & 6.25 & 0.0052 \\
Mgv-CK & 59755 & 1882 & 1921 & 6.56 & 0.0033 \\
\hline
\end{tabular}

the previous metagenomic projects on MGSs, termite gut, and cow rumen microbiome (Supplementary Table S9). Similar or even higher amounts of CNR genes from the GH family were found in our datasets. Some GH families, such GH 15 and GH 23, were not detected in the datasets of termite gut and cow rumen microbiome but were abundant in the datasets of this study. Therefore, although only CNR genes were involved in analysis, the diversity and abundance of GH genes from this study had clear advantages compared with that of other datasets.

\section{Abundance and Diversity of the Agarlytic Genes in MGSs}

Agarlytic genes belonging to GH 16, GH 50, GH 86, GH 96, and GH 117 were found in the datasets (Figure 2A). Furthermore, only the CNR agarlytic genes were involved in the follow-up analysis. GH 16 was the most abundant among these families. A total of 262 CNR agarlytic genes were observed in the control group, and the number of agarlytic genes was significantly increased to 588 in the enrichment group (Figure 2B). The number of beta-agarase and NABH genes rose from 224 and 34 to 469 and 116, respectively. In addition, the number of CNR $\mathrm{NABH}$ genes originating only from the datasets of enrichment group was $70 \%$ higher than those from all the previous reports according to the CAZy database (Supplementary Table S9).

The corresponding amino acid sequences of the CNR agarlytic genes were aligned in the NCBI nr database, and the best identities are shown in Figure 2C. The main distribution range was from $45 \%$ to $80 \%$. This result suggested that most of these agarlytic genes in MGSs are unreported.

\section{Recombinant Agarase Activity}

The optimum temperature of these agarase genes was predicted (Figure 3A). The values were mainly distributed between 40 and $50^{\circ} \mathrm{C}$. This finding indicated that most of the agarases from MGSs were mesophilic.

To utilize the agarase activity authentically in practical production, 30 genes (aga1-aga30) were expressed in the $E$. coli cells. Moreover, the effects of temperature and $\mathrm{pH}$ were determined (Supplementary Figures S5, S6). A total of 21 genes out of 30 genes were detected with activity (Figure 3B). Moreover, as predicted, the optimum temperatures were mainly 40 and $50^{\circ} \mathrm{C}$. Crude Aga 2 was detected with the highest specific activity (1.2 U/mg) among these agarases. Furthermore, a few extremozymes were found. The optimum temperatures for Aga28 and Aga29 were 30 and $70^{\circ} \mathrm{C}$, respectively. As another example, Aga13 possessed the optimum $\mathrm{pH}(\mathrm{pH}$ 5.0). This $\mathrm{pH}$ was lower than those of the others.

\section{Agarose-Degrading Characteristics of Aga2}

The agarose degradation characteristics of Aga2 were further studied after purification because of the highest crude enzyme activity of Aga2. The amino acid sequence of Aga2, which belonged to GH 16 family, showed $61.8 \%$ identity with the $\beta$-agarase from Bacteroides sartorii (WP_025018818.1). The MW of Aga2 was $\sim 37 \mathrm{kDa}$ according to SDS-PAGE. A clean zone was observed at the position of Aga2 after activity staining (Figure 4A). The optimum $\mathrm{pH}(7.0)$ and temperature $\left(50^{\circ} \mathrm{C}\right)$ of the purified Aga2 (Figures 4B,C) were in agreement with the previous results of the crude Aga2 (Figure 3B). The specific activity of Aga2 was $234.05 \mathrm{U} / \mathrm{mg}$ in the optimum conditions mentioned above. Aga2 was sensitive to $\mathrm{pH}$ and was only stable at a $\mathrm{pH}$ level of 7.0 (Figure 4B). Moreover, the Aga2 activity retained more than $85 \%$ after the preincubation at $20-40^{\circ} \mathrm{C}$ (Figure 4C). Aga2 resisted most chemical agents, including $\mathrm{NaCl}$, EDTA-2Na, and metal ions (except $\mathrm{Cu}^{2+}, \mathrm{Ni}^{2+}$, and $\mathrm{Ag}^{+}$). DTT and beta-Me increased the Aga2 activity moderately. However, most of the activity was lost with $1 \%$ and $10 \%$ SDS (Figure 4D).

\section{Analysis of the Signal Peptides of the Agarlytic Enzymes in MGSs}

Subsequently, the signal peptides of CNR agarases were analyzed using the SignalP script (Figure 5A). A total of $60.8 \%$ of the agarases were predicted without a signal peptide (Figure 5C). This result indicated that most of the agarases from MGSs cannot be transported out of the cell. Furthermore, the intracellular agarase activity $(1.31 \mathrm{mg} / \mathrm{mL})$ was significantly higher than the extracellular agarase activity $(0.61 \mathrm{mg} / \mathrm{mL}, p=0.014)$ in MGSs. This finding verified the possibility that more agarases remained in the cells of the microorganisms (Figure 5D).

To investigate whether this result is exclusive to our datasets or a general phenomenon, 424 agarases were selected as the reference set from the NCBI nr database to predict the signal peptide. The agarase ratio with signal peptides to that without signal peptides in the reference set (1.83) was significantly higher than those in the enrichment and control groups (0.65 and 0.49, respectively) (Figures 5B,C).

\section{Recovery of the Draft Genomes From the MGS Datasets}

Sequences from our datasets were binned and assembled into six draft genomes, which were named as Clusters 1-6 (Supplementary Tables S10, S11). According to the taxonomic annotation results (Supplementary Table S11), Clusters 1, 3, 5, and 6 were annotated as Desulfuromonas sp., Treponema sp., Ignavibacteriales spp., and Polyangiaceae spp., respectively. Meanwhile, Clusters 2 and 4 were potential new species. The accuracy of binning and assembly was verified by a 15-mer frequency distribution and the relationship between the GC content and sequence depth (Supplementary Figure S7). The result showed that the relationship between the GC content and sequence depth basically fitted the normal distribution, and the distribution 15-mer frequency accorded with the 

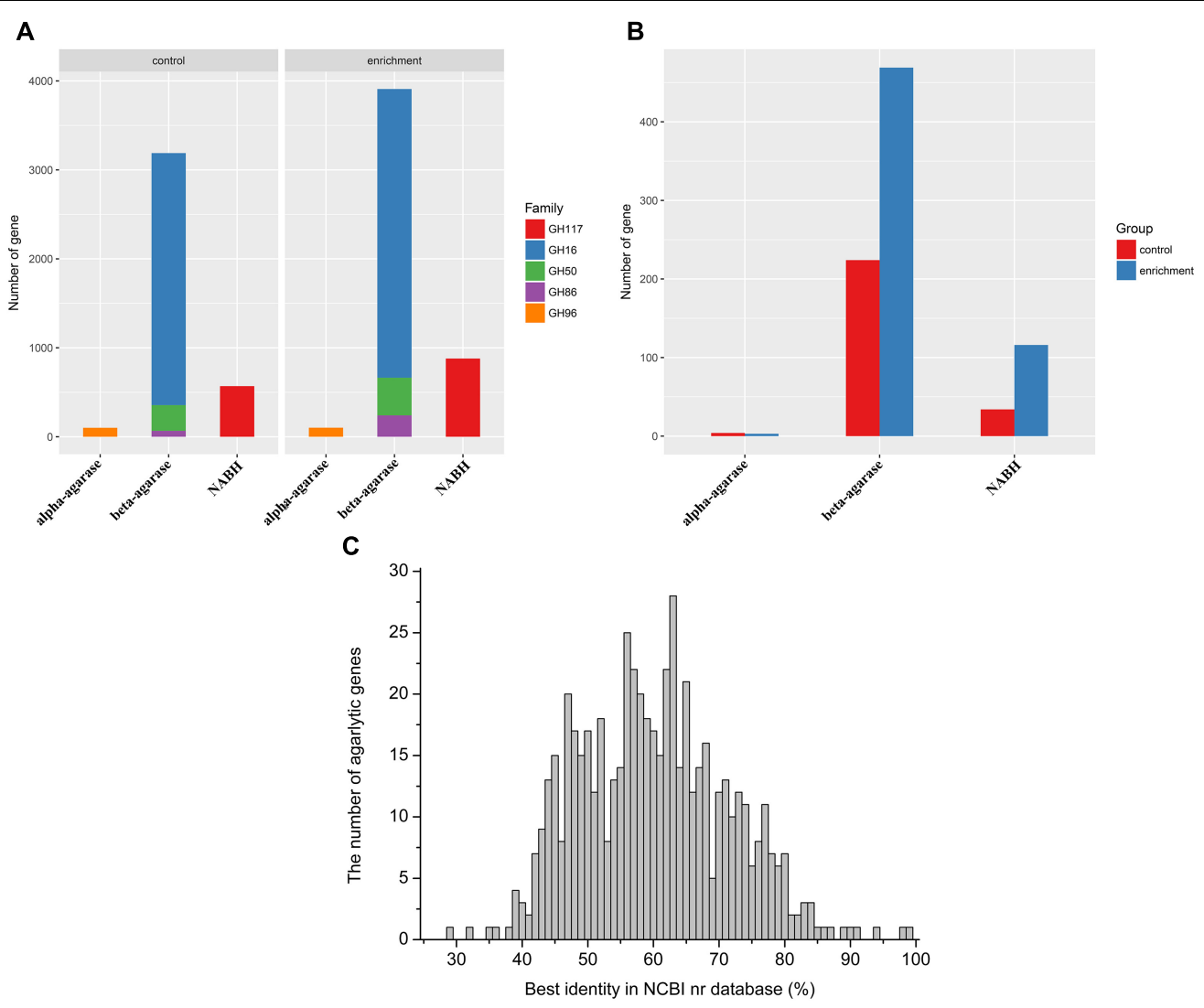

FIGURE 2 | Abundance and novelty of agarlytic genes in the MGS datasets. (A) The number of all agarlytic genes found in the datasets (NABH, $\alpha-1,3$-neoagarobiose hydrolase). (B) The number of complete and nonredundant (CNR) agarlytic genes in the datasets. (C) The distribution of the best identity of the corresponding amino acid sequences of the agarlytic genes in the NCBI nr database.
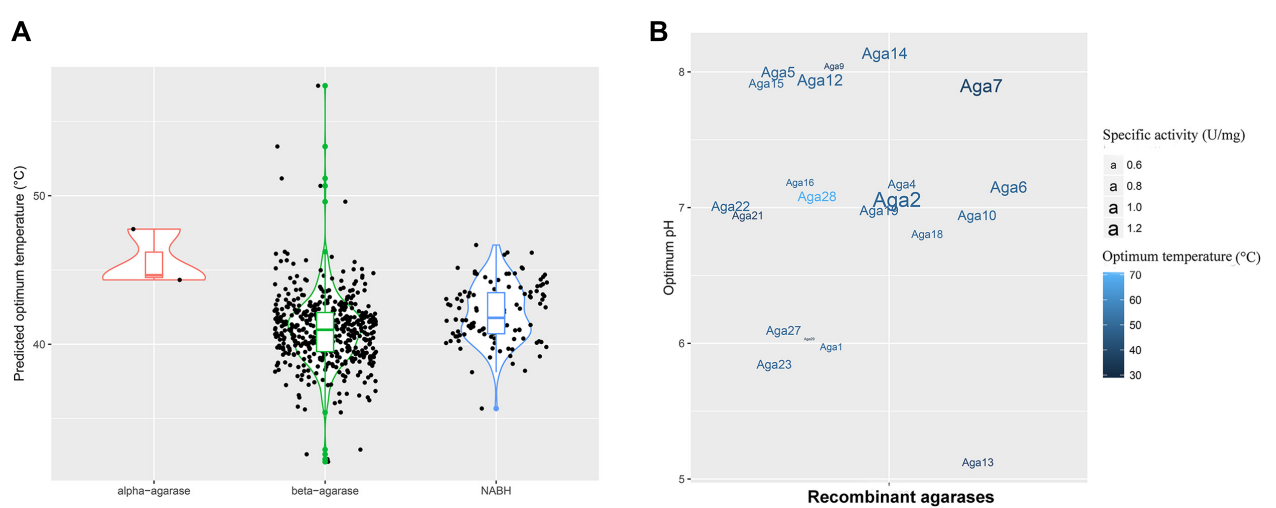

FIGURE 3 | Enzyme properties of agarases in the MGS datasets. (A) Density curve of the predicted optimum temperatures of the agarase in the MGS datasets. (B) The effects of $\mathrm{pH}$ and temperature on the activities of crude Aga1-Aga30. The color shades correspond to the optimum temperatures, and the text sizes stand for the specific activities of agarases.

poisson distribution, indicating a relatively high accuracy of these clusters. All these clusters lacked the reported reference genomes and were firstly reported. Clusters 2 and 4 were potential new species because the proteins in these genomes lack the concentrated BLAST hits in the NCBI nr database (Supplementary Table S11).

\section{Polysaccharide Degradation in the Draft Genomes}

All the six clusters were found with many genes related to glycometabolism (Figure 6A). Cluster 4 had the most genes for carbohydrate metabolism among these draft genomes according to the annotation results of KEGG database (Supplementary 


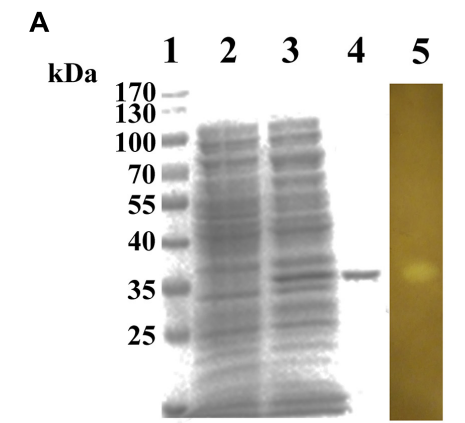

C

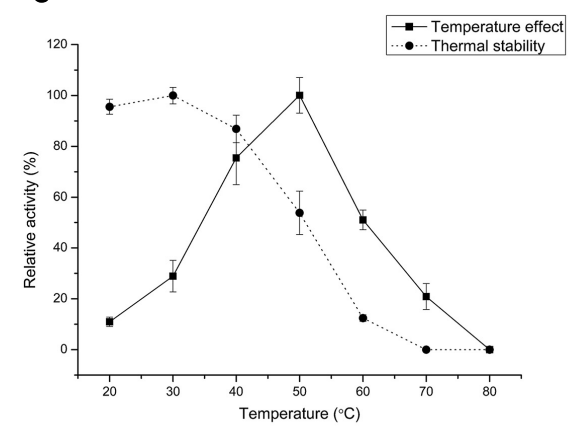

B

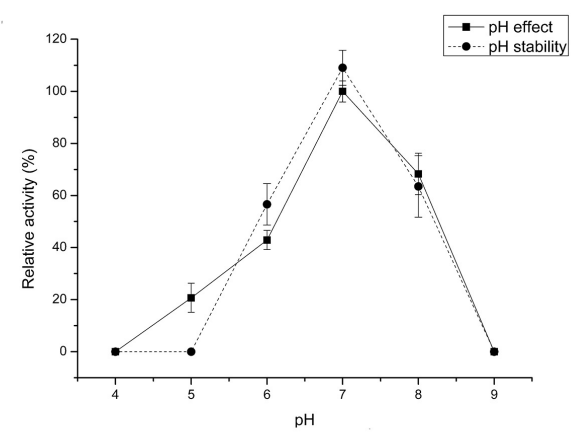

D

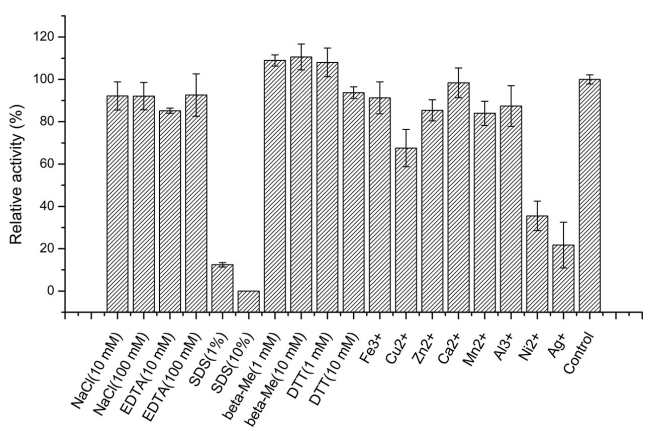

FIGURE 4 | Agarose-degrading characteristics of purified Aga2. (A) SDS-PAGE result. Lanes 1-5 stand for the standard MWs of proteins, supernatant of the cell disruption liquid with and without isopropyl $\beta$-D-thiogalactoside (IPTG) induction, purified Aga2, and the result of Aga2 activity staining. (B) The effects of pH on the activity and pH stability of purified Aga2. (C) The temperature effects on the activity and thermal stability of purified Aga2. (D) The effects of chemical agents on the activity of purified Aga2 (SDS, sodium dodecyl sulfate; DTT, dithiothreitol; beta-Me, beta-mercaptoethanol).

Figure S8). Meanwhile, most GH genes were found in Cluster 4 (Figure 6A). All these clusters possessed the degrading abilities of important polysaccharides, including starch, chitin, xylan, and cellulose (Figure 6B). Cluster 4 also had the most wide-ranging degrading ability for polysaccharides (Figure 6B).

Three clusters, namely, Clusters 4, 5, and 6, were found to harbor agarlytic genes. The locations of these enzymes were predicted (Supplementary Table S12). Most of the agarases in these genomes were located in the cytoplasm and had no signal peptides. This finding was in line with the analysis of the signal peptides in the datasets. Cluster 4 had the most CNR genes for utilizing agarose (Supplementary Table S12). Therefore, the agarose metabolism pathways of this cluster were speculated based on the annotation results from the CAZy and KEGG databases (Figure 6). The only agarase in Cluster 4 with signal peptide was transported outside the cell to cut the agarose into NAs that were subsequently transported into cells with the other NAs produced by the agarases in the environment. Agarases in the cytoplasm acted on the NAs to produce oligosaccharides with low MW. $\beta$-D-Galactose and 1,6anhydro- $\beta$-D-galactose (AHG) were produced by NABH. $\beta$-DGalactose was isomerized to $\alpha$-galactose by galM, an aldose 1-epimerase. $\alpha$-Galactose is further metabolized into glycolysis through the Leloir pathway. The metabolic pathway of AHG was absent in these clusters.

\section{DISCUSSION}

The organic carbon content in MGSs was studied in former studies. Bouillon et al. (2003) proved that the organic carbon content in India's MGSs was highly variable with a content of $0.6-31.7 \%$ dry weight. Their finding showed that this condition could affect the carbon dynamics in mangrove ecosystems (Bouillon et al., 2003). Donato et al. (2011) investigated 25 mangroves across of the Indo-Pacific region, and their results showed that an average $1,023 \mathrm{mg}$ carbon per hectare was contained in mangroves, and these mangroves are among the most carbon-rich forests in the tropics. Kenyan mangroves were investigated with an estimated belowground carbon of $69.41 \mathrm{t}$ (Gress et al., 2017). Kelleway et al. (2016) found that the 70-year mangroves contained approximately $12-15 \mathrm{~kg} / \mathrm{m}^{2}$ aboveground biomass and $20-30 \mathrm{~kg} / \mathrm{m}^{2}$ underground carbon. Therefore, mangroves serve as enormous warehouses for both aboveground and underground carbon. The genes for carbon cycling have been investigated by metagenomics sequencing (Andreote et al., 2012). Polysaccharides, such as cellulose, agarose, starch, and xylan, were important carbon forms in MGSs. Thus, MGSs are ideal environment for the enrichment of bacteria carrying polysaccharide-degrading genes. However, the abundance of genes responsible for polysaccharide degradation, which is the first step for carbon cycling and biofuel production, has rarely been comprehensively studied in early works. In former studies 

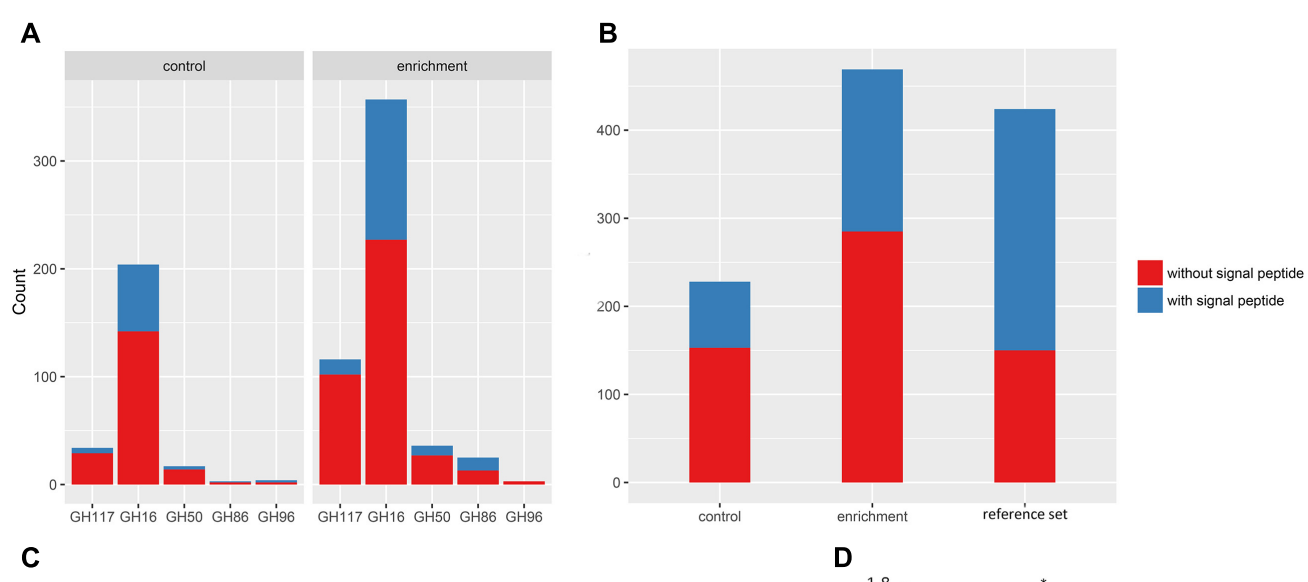

\begin{tabular}{cccc}
\hline Group & Number & Signal peptide & $\begin{array}{c}\text { Ratio } \\
\text { (with:without) }\end{array}$ \\
\hline enrichment & 184 & with & 0.65 \\
enrichment & 285 & without & \\
control & 75 & with & 0.49 \\
control & 153 & without & \\
reference set & 274 & with & 1.83 \\
reference set & 150 & without & \\
\hline
\end{tabular}

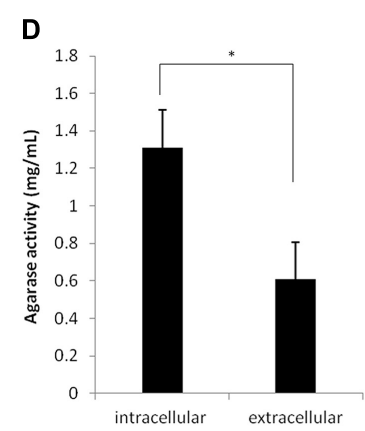

FIGURE 5 | Analysis of agarase signal peptides in the MGS datasets. (A) Signal peptide statistics of agarase in each GH family. (B) The signal peptide statistics of agarases in different datasets. (C) The ratio of agarases with signal peptides to those without signal peptides. (D) Extracellular and intracellular agarase activities in MGSs.

on MGSs by metagenomics sequencing, 454 pyrosequencing was the most frequently used method (Andreote et al., 2012; Thompson et al., 2013; Alzubaidy et al., 2016), and the relatively long reads from this strategy can improve the assembly effect. Thompson et al. (2013) investigated polysaccharide-degrading genes in Brazilian mangroves. Many cellulolytic genes and sequences, including cellulases, hemicellulases, carbohydratebinding domains, dockerins, and cohesins were detected, indicating that the microbiome in MGSs harbor all of the molecular tools for cellulose degradation and is an ideal source for cellulolytic genes. However, only 1,269,282 raw reads $(\sim 1.008 \mathrm{~Gb})$ for two samples were obtained in that study. For extremely complex microbial community in MGSs, this data size does not cover the majority of genetic information. Therefore, the real capacity of polysaccharide-degrading genes in MGSs can be more abundant than the data in that work. In the current study, we considerably increased the sequencing data to $\sim 300 \mathrm{~Gb}$, which is approximately a 100 - to 1000 -fold larger than those in previous reports. The abundance and completeness of the genes sharply increased with data size in our work.

Few studies have reported on agarases from MGSs. Shome et al. (2000) isolated the agar and agarose-degrading bacterium Alteromonas spp. from 1000 soil samples collected from various mangroves located in South Andaman. This bacterium can produce an extra-cellular exo-acting agarase whose optimum $\mathrm{pH}$ and temperature are $6.0-9.0$ and $25-37^{\circ} \mathrm{C}$, respectively (Shome et al., 2000). Mai et al. (2016) constructed a fosmid metagenomic library containing $\sim 3.0 \mathrm{~Gb}$ of genetic sequences using eDNA from MGSs, and a $\beta$-agarase belonging to GH 16 has been isolated from the library by functional screening. The optimum $\mathrm{pH}$ and temperature of the agarase were 7.0 and $50^{\circ} \mathrm{C}$, respectively, and this agarase was the first to be isolated from a metagenomics library of MGSs in 2016 (Mai et al., 2016). Agarases from other GH families have not been reported. In the current study, numerous agarlytic genes belonging to $\mathrm{GH} 16, \mathrm{GH} 50, \mathrm{GH} 86, \mathrm{GH}$ 96 , and GH 117 were discovered by deep sequencing. To improve the accuracy of our investigation, we only analyzed the CNR ORFs to discover the abundance and diversity of the agarlytic genes in MGSs. The results (Supplementary Table S9) showed that $204,17,3,4$, and $34 \mathrm{CNR}$ agarase genes belonged to $\mathrm{GH}$ 16, GH 50, GH 86, GH 96, and GH 117 were found in control group, respectively. Furthermore, 409, 36, 25, 3, and 116 CNR agarase genes belonged to $\mathrm{GH} 16, \mathrm{GH} 50, \mathrm{GH} 86, \mathrm{GH} 96$, and GH 117 were found in enrichment group, respectively. Obvious differences in the capacity and diversity of agarlytic genes were observed between a former study on MGSs (Thompson et al., 2013) and our work because no genes belonged to GH 50, GH 86, GH 96, and GH 117 in that study (Supplementary Table S9). Therefore, the capacity and diversity of agarlytic genes in MGSs microbiome is very abundant and seriously underestimated in former mangrove investigation (Thompson et al., 2013).

Agarlytic genes are not the only gene class that is abundant and diverse in our MGS datasets. Starch, cellulose, and xylan are also important and abundant polysaccharides in MGSs, and the 


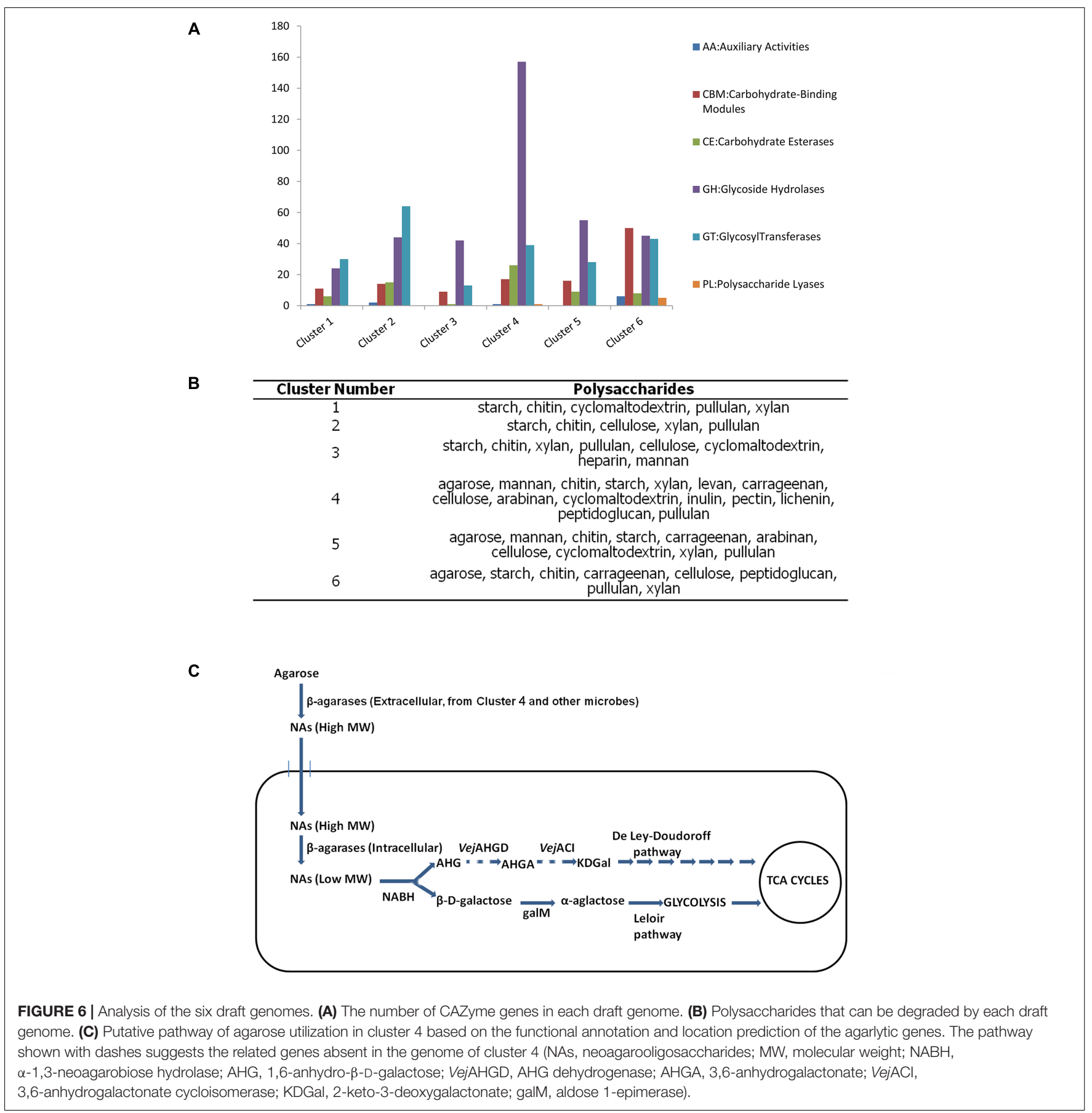

enzymes that degrade these polysaccharides were detected with great amount in our MGS datasets. Amylases mainly belong to GH 13 (Andrej et al., 2010; Majzlová et al., 2013; Peng et al., 2013; Štefan et al., 2015), whereas cellulases are mostly grouped into GH 3, GH 6, GH 12, and GH 17 (Sandgren et al., 2005; Van et al., 2012; Lima et al., 2013; Tishkov et al., 2013; Zhao et al., 2013; Jennifer et al., 2016; Zhang et al., 2016; Uchiyama et al., 2017). Xylanases are classified into GH 10 and GH 11 (Beaugrand et al., 2004; Dumon et al., 2008; Zhang et al., 2011; Paës et al., 2012; Wang et al., 2012). Therefore, these GH families are crucial for polysaccharide degradation in nature. The number of each $\mathrm{GH}$ in termite gut and rumen microbiome, two proven warehouses for cellulolytic genes, is listed in Supplementary Table S9. In termite gut microbiome datasets, 69, 46, 14, and 48 genes belonging to $\mathrm{GH} 3, \mathrm{GH} 10, \mathrm{GH} 11$, and $\mathrm{GH} 13$ were found. In rumen microbiome datasets, 2844, 1025, 165, and 3442 genes belonging to GH 3, GH 10, GH 11, and GH 13 were identified (Supplementary Table S9). However, few GH 6, GH 12, and GH 17 genes were annotated in both datasets. Although the number of genes belonging to $\mathrm{GH} 3, \mathrm{GH} 10, \mathrm{GH} 11$, and $\mathrm{GH} 13$ were 
less in our MGSs datasets (both control and enrichment group) than that in rumen microbiome, the genes from these families in our MGS datasets were more abundant than those in termite gut datasets (Supplementary Table S9). The GH families that are not or rarely detected in termite gut and rumen microbiome, such as GH 6, GH 12, GH 17, GH 46, GH 47, GH 102, and GH 103 , were abundant in our MGS datasets. Compared with former datasets, our results showed high diversity and capacity of not only agarlytic CNR genes but also that of nearly all CNR GH family genes in MGS microbiome.

Interestingly, few agarlytic bacteria and genes were isolated from the MGS microbiome but with high capacity of agarases proven by our data. Functional screening strategy is usually employed for the isolation of functional genes from environmental samples for both pure culturing and metagenomics library construction. Signal peptides are contiguous amino acid sequences that are mostly present in proteins and directs protein localization (von Heijne, 1990). Given the lack of membranous organelles in bacteria, bacterial proteins containing signal peptides must be transported extracellularly or to the cell membrane. Thus, functional screening-based strategy heavily depends on the signal peptide in enzymes because no significant phenomenon can be observed during functional screening if the protein cannot transport extracellularly. Therefore, we analyzed signal peptide within agarases from MGS datasets. Normally, agarases must be transported to or outside the cell membrane to play a role because high MW agarose can barely be transported into cells. Therefore, $64.6 \%$ of the agarases from the reference set were predicted to possess signal peptides (Figure 5C). However, the proportion of agarase with signal peptides was significantly lower in the mangrove datasets (39.4 and 32.9\% for enrichment and control group, respectively) than that in the reference set. This result may explain the reason of rare agarases isolated from MGSs. The deficiency of signal peptide in most of the agarases from MGSs caused difficulty in discovering agarlytic genes through functional screening. Moreover, this observation suggested that only a small portion of agarases are carried externally from the cell to degrade agarose into low-MW oligosaccharides that can be transported into cells. The rest of the agarases are retained in the cells to degrade entering oligosaccharides, such as the pathway of agarose degradation in Cluster 4. Using this strategy, a bacterium can highly utilize the oligosaccharides produced by other agarlytic microbes, and the resources and energy for signal peptide recognition, translocation, and resection of most agarases can be conserved. Therefore, utilizing agarose in MGSs may be collaborative and economical.

Full-length of ORFs is difficult to obtain using sequencingbased metagenomics, Especially in samples containing complex microbial community. Brulc et al. (2009) investigated the cellulolytic genes in rumen microbiome and found that CBM domains that are under-represented in cellulolytic genes are often absent, which caused incomplete ORFs in the datasets. Similar results were obtained in the microbiome of Tammar wallaby by Pope and Moran (2010). Hess et al. (2011) stated that sequence-based metagenomic discovery of complete genes from environmental samples is limited by the microbial species complexity of most environments and the consequent rarity of full-length genes in lowcoverage metagenomic assemblies. Therefore, increasing the environmental samples with complex microbial community is important to improve the completeness of ORFs. Unfortunately, MGSs are among complex environmental samples. Hence, fulllength ORFs were rarely obtained in former metagenomics sequencing studies on MGSs. The incomplete ORFs will weaken the practical value of metagenomics studies because of problems in protein expression. Unlike the case for these metagenomics studies, we aimed to obtain complete agarase genes for heterologous expressions to identify the real activities of the enzymes for possible production applications by substantially increasing the depth of sequencing. Consequently, 469 and 228 CNR agarase genes were obtained in the enrichment and control groups, respectively. A total of 21 expressed CNR agarase genes were detected with the ability to degrade agarose. Some enzymes ( 9 out of the 30 enzymes) lacked this activity because of possibly low expression levels, inclusion body formation, and inappropriate conditions for protein folding and functioning. Among these agarases, Aga28 and Aga29 could work optimally at extreme temperatures $\left(30\right.$ and $70^{\circ} \mathrm{C}$, respectively). Aga2, which exhibits the highest crude enzyme activity of agarose degradation among the agarases expressed in this study, was purified. We found that Aga2 demonstrates a $\mathrm{MW}$ of $37 \mathrm{kDa}$ and a high specific activity of $234.05 \mathrm{U} / \mathrm{mg}$. Former review (Ting and Moo, 2010) summarized the properties of existing recombinant agarases and showed that except for two recombinant agarase (agaA and agaB) with extremely high activity, the MWs and specific activities of these agarase mainly ranged from 30 to $146 \mathrm{kDa}$ and from 16.4 to $398 \mathrm{U} / \mathrm{mg}$, respectively, which demonstrated that Aga2 possessed a lower MW and a higher specific activity among most recombinant agarases, indicating Aga2 could efficiently degrade agarose with less consumption of amino acids. In addition, Aga 2 is resistant to temperature change from 20 to $40^{\circ} \mathrm{C}$ and many chemical agents. In our parallel study (Di et al., 2018), Agal was renamed AgaM1 to avoid duplication and was further purified for the analysis of thermodynamic and kinetic properties, and the results showed the purified Agal possessed extraordinary thermo and $\mathrm{pH}$ stabilities, even better than Aga2. In fact, the weak stability against temperatures and pHs limited the application of many agarases (Shome et al., 2000; Fu et al., 2009; Dong et al., 2016; Zhu et al., 2016; Jung et al., 2017), and thus, these mangrovederived enzymes with excellent stabilities were good options for industrial production with complex and varying conditions. Given that most of these CNR agarase genes are unreported, the agarases in MGSs are not only abundant in number, but also possess sequence novelty and outstanding enzymatic characteristics.

Aga2 was grouped in the GH 16 family, and the degradation end-products of agarose by the agarase of GH 16 family are neoagarotetraose (NA4) and neoagarohexaose (NA6) according to CAZy database and former studies (Ohta et al., 2004; Dong et al., 2016). The biological activities of NA4 and NA6 have been confirmed by many works. Wang et al. (2017) demonstrated that NAs with different degrees of polymerization, including NA4 and 
NA6, possess anti-inflammatory activity in lipopolysaccharidesstimulated macrophages. NA4 and NA6 exhibit antibacterial activity against several Gram-negative and -positive bacteria (Hong et al., 2017a). NA4 and NA6 can also induce adiponectin production and effectively suppress obesity and obesity-related metabolic syndromes in obese mice (Hong et al., 2017b). Hou et al. (2014) demonstrated that treatment with neoagarooligosaccharide with 4-8 degrees of polymerization can reduce the consumption of fruit weight, titratable acid, and vitamin C contents of cherry tomatoes. Moreover, the safety of NA4 and NA6 has been confirmed by previous genotoxicity test (Hong et al., 2017c). Therefore, Aga2 expressed in this study possesses great potential in food and pharmaceutical industries. Furthermore, a great number of NABH genes belonging to $\mathrm{GH}$ 117 family were found in our datasets. NABH is key to hydrolyze NA2 into L-AHG and D-galactose, two valuable fermentable monosaccharides, by cleaving $\alpha-1,3$ linkage (Watanabe et al., 2017). D-Galactose is an important precursor of bio-fuel, such as ethanol and butanol (Chen and Dixon, 2007; Ha et al., 2015; Hyungyu et al., 2015), as well as L-AHG (Yun et al., 2015b). Besides, a recent Japanese potent study showed that L-AHG could inhibit melanin production, indicating the whitening ability of LAHG (K. Kirimura and T. Koide, Japan patent JP2013-247884). The substantial NABH genes obtained in this study can provide more choices to hydrolyze NA2 for the production of L-AHG and $\mathrm{D}$-galactose. Therefore, above enzymes possess great potential in food, pharmaceutical, cosmetic, and bio-fuel industries.

Genome sequences are crucial to study metabolic mechanism and pathway in nonculturable bacteria, which comprise more than $90 \%$ of microorganisms in the world. Unfortunately, the data sizes of previous studies are insufficient to assemble genomes. Our study was the first to attempt to assemble the genomes from MGS datasets. All of these draft genomes remained unreported and exhibited many CAZyme genes and degrading ability for several polysaccharides. Among these draft genomes, clusters 1, 3, 5, and 6 were annotated as Desulfuromonas genus, Treponema genus, Ignavibacteriales order, and Polyangiaceae family. Desulfuromonas is closely related to sulfur reduction, acetate oxidization, and $\mathrm{Fe}$ (III) reduction (Pfennig and Biebl, 1976; Roden and Lovley, 1993; Coates et al., 1995). Bacteria in the Treponema genus are often pathogens of human and animals (Harris et al., 1972; Fraser et al., 1998; Handsfield, 2004). Ignavibacteriales order is reported with the function of $\mathrm{CO}_{2}$ fixation (Iino et al., 2010). Polyangiaceae family is the only family of myxobacteria that has only included cellulose-degrading strains (Garcia and Müller, 2014). Clusters 2 and 4 are potential new species, and Cluster 4 harbored complex degrading-ability for most polysaccharides, including starch, chitin, cellulose, pectin, agarose, and so on. This result indicated that several new and unknown microbial resources for the degradation of complex polysaccharides may remain unexplored in MGS microbiome. The metabolic pathway of agarose was speculated in Cluster 4 . However, the enzymes for AHG metabolism that possibly include AHG dehydrogenase (VejAHGD) and 3,6-anhydrogalactonate (AHGA) cycloisomerase (VejACI) (Choi et al., 2011, 2013; Yun et al., 2015a) were not detected probably because of incomplete assembly. Iverson et al. (2012) assembled the genome of an uncultured marine group II Euryarchaeote through an ocean water metagenomics dataset with about threefold mate-pair reads relative to that in the Global Ocean Sampling database (Rusch et al., 2007). The high coverage of database resulted in a nearly closed genome of marine group II Euryarchaeote. Luo et al. (2012) performed the genomic assemblies using the datasets derived from the microbial community in freshwater, soil, and in silico simulations. The results revealed that the genome of a single genotype or species can be accurately recovered and assembled using a $20 \times$ coverage metagenomics database from environmental samples with complex microbial community (Luo et al., 2012), suggesting that the integrity of a genome assembly relies heavily on sequencing coverage. However, even the large data size in our study only reluctantly supported the genome assembly and could not extract sufficient reads, which caused low-coverage and fragmented genomes. This result also indicates that enormous genetic resources, including polysaccharidedegrading genes, were uncovered in the MGS microbiome. We believe that with further studies, massive datasets that are similar in size to that of human gut microbiota will be generated. Using these terabase-level data, complete and valuable genomes and several undiscovered genomes and pathways in MGSs will be obtained.

In summary, this study demonstrated the advantages of MGS microbiome in agarlytic gene isolation from the aspects of the capacity, diversity, activity, novelty, signal peptide and related unculturable genomes, enlarged the routine roles of mangroves, and provided insight into the potential of MGSs as potential repertories not only of the agarlytic genes but almost all of the CAZyme family genes.

\section{AUTHOR CONTRIBUTIONS}

WQ and RZ designed this study. WQ and WD performed the experiences. WQ, DL, ZZ, and RZ analyzed the sequencing data. WQ, BG, and RZ wrote the paper. All the authors have read and approved the final manuscript.

\section{FUNDING}

This research was supported by grants from the Scientific Research Foundation of Third Institute of Oceanography, SOA (Grant No. 2016038); Public Science and Technology Research Funds Projects of Ocean (Grant No. 2015050262); the Natural Science Foundation of Fujian Province of China (Grant No. 2016J01160); Xiamen Ocean Economic Innovation and Development Demonstration Project (Grant No. 16PZP001SF16).

\section{SUPPLEMENTARY MATERIAL}

The Supplementary Material for this article can be found online at: https://www.frontiersin.org/articles/10.3389/fmicb. 2018.01864/full\#supplementary-material 


\section{REFERENCES}

Alneberg, J., Bjarnason, B. S., De Bruijn, I., Schirmer, M., Quick, J., Ijaz, U. Z., et al. (2014). Binning metagenomic contigs by coverage and composition. Nat. Methods 11, 1144-1146. doi: 10.1038/nmeth.3103

Alzubaidy, H., Essack, M., Malas, T. B., Bokhari, A., Motwalli, O., Kamanu, F. K., et al. (2016). Rhizosphere microbiome metagenomics of gray mangroves (Avicennia marina) in the Red Sea. Gene 576, 626-636. doi: 10.1016/j.gene.2015. 10.032

Anantharaman, K., Breier, J. A., and Dick, G. J. (2016). Metagenomic resolution of microbial functions in deep-sea hydrothermal plumes across the Eastern Lau Spreading Center. ISME J. 10, 225-239. doi: 10.1038/ismej.2015.81

Andrej, G., Katarína, M., Viera, H., Barbora, V., and Štefan, J. (2010). Tyrosine 39 of GH13 $\alpha$-amylase from Thermococcus hydrothermalis contributes to its thermostability. Biologia 65, 408-415.

Andreote, F. D., Jiménez, D. J., Chaves, D., Dias, A. C. F., Luvizotto, D. M., DiniAndreote, F., et al. (2012). The microbiome of Brazilian mangrove sediments as revealed by metagenomics. PLoS One 7:e38600. doi: 10.1371/journal.pone. 0038600

Bankevich, A., Nurk, S., Antipov, D., Gurevich, A. A., Dvorkin, M., Kulikov, A. S., et al. (2012). SPAdes: a new genome assembly algorithm and its applications to single-cell sequencing. J. Comput. Biol. 19, 455-477. doi: 10.1089/cmb.2012. 0021

Beaugrand, J., Chambat, G., Wong, V. W., Goubet, F., Rémond, C., Paës, G., et al. (2004). Impact and efficiency of GH10 and GH11 thermostable endoxylanases on wheat bran and alkali-extractable arabinoxylans. Carbohydr. Res. 339, 25292540. doi: 10.1016/j.carres.2004.08.012

Bharathkumar, S., Paul, D., and Nair, S. (2008). Microbial diversity of culturable heterotrophs in the rhizosphere of salt marsh grass, Porteresia coarctata (Tateoka) in a mangrove ecosystem. J. Basic Microbiol. 48, 10-15. doi: 10.1002/ jobm.200700282

Bouillon, S., Dahdouh-Guebas, F., Rao, A. V. V. S., Koedam, N., and Dehairs, F. (2003). Sources of organic carbon in mangrove sediments: variability and possible ecological implications. Hydrobiologia 495, 33-39. doi: 10.1023/A: 1025411506526

Brulc, J. M., Antonopoulos, D. A., Miller, M. E., Wilson, M. K., Yannarell, A. C., Dinsdale, E. A., et al. (2009). Gene-centric metagenomics of the fiber-adherent bovine rumen microbiome reveals forage specific glycoside hydrolases. Proc. Natl. Acad. Sci. U.S.A. 106, 1948-1953. doi: 10.1073/pnas.0806191105

Caporaso, J. G., Kuczynski, J., Stombaugh, J., Bittinger, K., Bushman, F. D., Costello, E. K., et al. (2010). QIIME allows analysis of high-throughput community sequencing data. Nat. Methods 7, 335-336. doi: 10.1038/nmeth.f. 303

Chen, F., and Dixon, R. A. (2007). Lignin modification improves fermentable sugar yields for biofuel production. Nat. Biotechnol. 25, 759-761. doi: 10.1038/ nbt1316

Choi, I. G., Kim, K. H., Lee, S., and Yun, E. J. (2013). Novel 3, 6-anhydro galactonic acid cycloisomerase and use thereof. Pohang: Korea Patent.

Choi, I. G., Kim, K. H., Yun, E. J., and Lee, S. (2011). A novel 3, 6-anhydroL-galactose dehydrogenase to convert 3, 6-anhydro-L-galactose into 3, 6anhydrogalactonic acid. Pohang: Korea Patent.

Chu, Y., Yi, Z., Zeng, R., and Zhang, G. (2016). Predicting the optimum temperature of $\beta$-agarase based on the relative solvent accessibility of amino acids. J. Mol. Catal. B 129, 47-53. doi: 10.1016/j.molcatb.2016.04.005

Coates, J. D., Lonergan, D. J., Philips, E. J. P., Jenter, H., and Lovley, D. R. (1995). Desulfuromonas palmitatis sp. nov., a marine dissimilatory $\mathrm{Fe}(\mathrm{III})$ reducer that can oxidize long-chain fatty acids. Arch. Microbiol. 164, 406-413. doi: 10.1007/ BF02529738

D’Onofrio, A., Crawford, J. M., Stewart, E. J., Witt, K., Gavrish, E., Epstein, S., et al. (2010). Siderophores from neighboring organisms promote the growth of uncultured bacteria. Chem. Biol. 17, 254-264. doi: 10.1016/j.chembiol.2010. 02.010

Di, W., Qu, W., and Zeng, R. (2018). Cloning, expression, and characterization of thermal-stable and $\mathrm{pH}$-stable agarase from mangrove sediments. J. Basic Microbiol. 58, 302-309. doi: 10.1002/jobm.201700696

Dias, A. C. F., Andreote, F. D., Dini-Andreote, F., Lacava, P. T., Sá, A. L. B., Melo, I. S., et al. (2009). Diversity and biotechnological potential of culturable bacteria from Brazilian mangrove sediment. World J. Microbiol. Biotechnol. 25, 1305-1311. doi: 10.1007/s11274-009-0013-7
Donato, D. C., Kauffman, J. B., Murdiyarso, D., Kurnianto, S., Stidham, M., and Kanninen, M. (2011). Mangroves among the most carbon-rich forests in the tropics. Nat. Geosci. 4, 293-297. doi: 10.1038/ngeo1123

Dong, Q., Ruan, L., and Shi, H. (2016). A $\beta$-agarase with high $\mathrm{pH}$ stability from Flammeovirga sp. SJP92. Carbohydr. Res. 432, 1-8. doi: 10.1016/j.carres.2016. 05.002

Dumon, C., Varvak, A., Wall, M. A., Flint, J. E., Lewis, R. J., Lakey, J. H., et al. (2008). Engineering hyperthermostability into a GH11 xylanase is mediated by subtle changes to protein structure. J. Biol. Chem. 283, 22557-22564. doi: 10.1074/jbc.M800936200

Enoki, T., Okuda, S., Kudo, Y., Takashima, F., Sagawa, H., and Kato, I. (2010). Oligosaccharides from agar inhibit pro-inflammatory mediator release by inducing heme oxygenase 1. Biosci. Biotechnol. Biochem. 74, 766-770. doi: 10. 1271/bbb. 90803

Fan, W., Tang, Y., Qu, Y., Cao, F., and Huo, G. (2014). Infant formula supplemented with low protein and high carbohydrate alters the intestinal microbiota in neonatal SD rats. BMC Microbiol. 14:279. doi: 10.1186/s12866014-0279-2

Fraser, C. M., Norris, S. J., Weinstock, G. M., White, O., Sutton, G. G., Dodson, R., et al. (1998). Complete genome sequence of Treponema pallidum, the syphilis spirochete. Science 281, 375-388. doi: 10.1126/science.281.5375.375

Fu, X. T., Pan, C. H., Hong, L., and Sang, M. K. (2009). "Gene cloning,expression, and characterization of a $\beta$-Agarase,AgaB34,from Agarivorans albus YKW-34", in Proceedings of the International symposium on seafood processing technology and safety control system, Korea, 257.

Garcia, R., and Müller, R. (2014). The Family Polyangiaceae. Heidelberg: Springer Berlin Heidelberg. doi: 10.1007/978-3-642-39044-9_308

Girotto, S., Pizzi, C., and Comin, M. (2016). MetaProb: accurate metagenomic reads binning based on probabilistic sequence signatures. Bioinformatics 32, i567-i575. doi: 10.1093/bioinformatics/btw466

Gress, S. K., Huxham, M., Kairo, J. G., Mugi, L. M., and Briers, R. A. (2017). Evaluating, predicting and mapping belowground carbon stores in Kenyan mangroves. Glob. Change Biol. 23, 224-234. doi: 10.1111/gcb.13438

Ha, S. J., Wei, Q., Kim, S. R., Galazka, J. M., Cate, J., and Jin, Y. S. (2015). Cofermentation of cellobiose and galactose by an engineered Saccharomyces cerevisiae strain. Appl. Environ. Microbiol. 77, 5822-5825. doi: 10.1128/AEM. 05228- 11

Handsfield, H. H. (2004). Macrolide resistance in Treponema pallidum in the United States and Ireland - NEJM. N. Engl. J. Med. 351, 154-158. doi: 10.1056/ NEJMoa040216

Harris, D. L., Glock, R. D., Christensen, C. R., and Kinyon, J. M. (1972). Inoculation of pigs with Treponema hyodysenteriae (new species) and reproduction $f$ the disease. Vet. Med. Small Anim. Clin. 67, 61-64.

Harris, P. V., Welner, D., McFarland, K. C., Re, E., Navarro Poulsen, J. C., Brown, K., et al. (2010). Stimulation of lignocellulosic biomass hydrolysis by proteins of glycoside hydrolase family 61: structure and function of a large, enigmatic family. Biochemistry 49, 3305-3316. doi: 10.1021/bi100009p

Heinze, T., and Liebert, T. (2001). Unconventional methods in cellulose functionalization. Prog. Polym. Sci. 26, 1689-1762. doi: 10.1016/S0079-6700(01) 00022-3

Hess, M., Sczyrba, A., Egan, R., Kim, T. W., Chokhawala, H., Schroth, G., et al. (2011). Metagenomic discovery of biomass-degrading genes and genomes from cow rumen. Science 331, 463-467. doi: 10.1126/science.1200387

Hong, S. J., Lee, J. H., Kim, E. J., Yang, H. J., Chang, Y. K., Park, J. S., et al. (2017a). In vitro and in vivo investigation for biological activities of neoagarooligosaccharides prepared by hydrolyzing agar with $\beta$-agarase. Biotechnol. Bioprocess Eng. 22, 489-496. doi: 10.1007/s12257-017-0049-8

Hong, S. J., Lee, J. H., Kim, E. J., Yang, H. J., Park, J. S., and Hong, S. K. (2017b). Anti-obesity and anti-diabetic effect of neoagarooligosaccharides on high-fat diet-induced obesity in mice. Mar. Drugs 15:E90. doi: 10.3390/md15040090

Hong, S. J., Lee, J. H., Kim, E. J., Yang, H. J., Park, J. S., and Hong, S. K. (2017c). Toxicological evaluation of neoagarooligosaccharides prepared by enzymatic hydrolysis of agar. Regul. Toxicol. Pharmacol. 90, 9-21. doi: 10.1016/j.yrtph. 2017.08.001

Hou, Y., Gao, J., Gu, L., Wang, S., and Zeng, R. (2014). Effects of agarooligosaccharide treatment on postharvest quality of cherry tomatoes during cold storage. J. Food Process. Preserv. 39, 949-955. doi: 10.1111/jfpp. 12308 
Hu, B., Gong, Q., Wang, Y., Ma, Y., Li, J., and Yu, W. (2006). Prebiotic effects of neoagaro-oligosaccharides prepared by enzymatic hydrolysis of agarose. Anaerobe 12, 260-266. doi: 10.1016/j.anaerobe.2006.07.005

Huson, D. H., Mitra, S., Ruscheweyh, H. J., Weber, N., and Schuster, S. C. (2011). Integrative analysis of environmental sequences using MEGAN4. Genome Res. 21, 1552-1560. doi: 10.1101/gr.120618.111

Hyatt, D., Chen, G. L., LoCascio, P. F., Land, M. L., Larimer, F. W., and Hauser, L. J. (2010). Prodigal: prokaryotic gene recognition and translation initiation site identification. BMC Bioinformatics 11:119. doi: 10.1186/1471-2105-11-119

Hyungyu, L., Jaehyung, L., and Gyooyeol, J. (2015). Modular design of metabolic network for robust production of n-butanol from galactose-glucose mixtures. Biotechnol. Biofuels 8:137. doi: 10.1186/s13068-015-0327-7

Iino, T., Mori, K., Uchino, Y., Nakagawa, T., Harayama, S., and Suzuki, K. (2010). Ignavibacterium album gen. nov., sp. nov., a moderately thermophilic anaerobic bacterium isolated from microbial mats at a terrestrial hot spring and proposal of Ignavibacteria classis nov., for a novel lineage at the periphery of green sulfur bacteria. Int. J. Syst. Evol. Microbiol. 60(Pt 6), 1376-1382. doi: 10.1099/ijs.0. 012484-0

Iverson, V., Morris, R. M., Frazar, C. D., Berthiaume, C. T., Morales, R. L., and Armbrust, E. V. (2012). Untangling genomes from metagenomes: revealing an uncultured class of marine Euryarchaeota. Science 335, 587-590. doi: 10.1126/ science. 1212665

Jennifer, H., Wu, Y. W., Kai, D., Nicora, C. D., Aldrich, J. T., Dario, F., et al. (2016). Comparative community proteomics demonstrates the unexpected importance of actinobacterial glycoside hydrolase family 12 protein for crystalline cellulose hydrolysis. $m$ Bio 7:e01106-16.

Jeoh, T., Ishizawa, C. I., Davis, M. F., Himmel, M. E., Adney, W. S., and Johnson, D. K. (2007). Cellulase digestibility of pretreated biomass is limited by cellulose accessibility. Biotechnol. Bioeng. 98, 112-122. doi: 10.1002/bit.21408

Jiang, X. T., Peng, X., Deng, G. H., Sheng, H. F., Wang, Y., Zhou, H. W., et al. (2013). Illumina sequencing of $16 \mathrm{~S}$ rRNA tag revealed spatial variations of bacterial communities in a mangrove wetland. Microb. Ecol. 66, 96-104. doi: 10.1007/s00248-013-0238-8

Jung, S., Jeong, B. C., Hong, S. K., and Lee, C. R. (2017). Cloning, Expression, and Biochemical characterization of a novel acidic GH16 $\beta$-agarase, AgaJ11, from gayadomonas joobiniege G7. Appl. Biochem. Biotechnol. 181, 961-971. doi: 10.1007/s12010-016-2262-x

Kelleway, J. J., Saintilan, N., Macreadie, P. I., Skilbeck, C. G., Zawadzki, A., and Ralph, P. J. (2016). Seventy years of continuous encroachment substantially increases 'blue carbon' capacity as mangroves replace intertidal salt marshes. Glob. Change Biol. 22, 1097-1109. doi: 10.1111/gcb.13158

Kim, H. T., Lee, S., Kim, K. H., and Choi, I. G. (2012). The complete enzymatic saccharification of agarose and its application to simultaneous saccharification and fermentation of agarose for ethanol production. Bioresour. Technol. 107, 301-306. doi: 10.1016/j.biortech.2011.11.120

Kim, K. H., and Hong, J. (2001). Supercritical CO2 pretreatment of lignocellulose enhances enzymatic cellulose hydrolysis. Bioresour. Technol. 77, 139-144. doi: $10.1016 /$ S0960-8524(00)00147-4

Kuo, C. H., and Lee, C. K. (2009). Enhancement of enzymatic saccharification of cellulose by cellulose dissolution pretreatments. Carbohydr. Polym. 77, 41-46. doi: 10.1016/j.carbpol.2008.12.003

Langston, J. A., Shaghasi, T., Abbate, E., Xu, F., Vlasenko, E., and Sweeney, M. D. (2011). Oxidoreductive cellulose depolymerization by the enzymes cellobiose dehydrogenase and glycoside hydrolase 61. Appl. Environ. Microbiol. 77, 70077015. doi: 10.1128/AEM.05815-11

Lengeler, J. W., Drews, G., and Schlegel, H. G. (2009). Chapter 30. Ecophysiology and Ecological Niches of Prokaryotes. Hoboken, NJ: Blackwell Science Ltd.

Leung, H. C., Yiu, S. M., Yang, B., Peng, Y., Wang, Y., Liu, Z., et al. (2011). A robust and accurate binning algorithm for metagenomic sequences with arbitrary species abundance ratio. Bioinformatics 27, 1489-1495. doi: 10.1093/ bioinformatics/btr186

Li, D., Liu, C. M., Luo, R., Sadakane, K., and Lam, T. W. (2015). MEGAHIT: an ultra-fast single-node solution for large and complex metagenomics assembly via succinct de Bruijn graph. Bioinformatics 31, 1674-1676. doi: 10.1093/ bioinformatics/btv033

Li, J., Jia, H., Cai, X., Zhong, H., Feng, Q., Sunagawa, S., et al. (2014). An integrated catalog of reference genes in the human gut microbiome. Nat. Biotechnol. 32, 834-841. doi: 10.1038/nbt.2942
Li, R., Li, Y., Kristiansen, K., and Wang, J. (2008). SOAP: short oligonucleotide alignment program. Bioinformatics 24, 713-714. doi: 10.1093/bioinformatics/ btn025

Lima, M. A., Oliveiraneto, M., Kadowaki, M. A., Rosseto, F. R., Prates, E. T., Squina, F. M., et al. (2013). Aspergillus niger $\beta$-glucosidase has a cellulase-like tadpole molecular shape: insights into glycoside hydrolase family 3 (GH3) $\beta$-glucosidase structure and function. J. Biol. Chem. 288, 32991-33005. doi: 10.1074/jbc.M113. 479279

Liu, N., Yan, X., Zhang, M., Xie, L., Wang, Q., Huang, Y., et al. (2011). Microbiome of fungus-growing termites: a new reservoir for lignocellulase genes. Appl. Environ. Microbiol. 77, 48-56. doi: 10.1128/AEM.01521-10

Luo, C., Tsementzi, D., Kyrpides, N. C., and Konstantinidis, K. T. (2012). Individual genome assembly from complex community short-read metagenomic datasets. ISME J. 6, 898-901. doi: 10.1038/ismej.2011.147

Lynd, L. R., Weimer, P. J., van Zyl, W. H., and Pretorius, I. S. (2002). Microbial cellulose utilization: fundamentals and biotechnology. Microbiol. Mol. Biol. Rev. 66, 506-577. doi: 10.1128/MMBR.66.3.506-577.2002

Mai, Z., Su, H., and Si, Z. (2016). Isolation and characterization of a glycosyl hydrolase family $16 \beta$-agarase from a mangrove soil metagenomic library. Int. J. Mol. Sci. 17:E1360. doi: 10.3390/ijms17081360

Majzlová, K., Pukajová, Z., and Janeěek, S. (2013). Tracing the evolution of the $\alpha$-amylase subfamily GH13_36 covering the amylolytic enzymes intermediate between oligo-1,6-glucosidases and neopullulanases. Carbohydr. Res. 367, 4857. doi: 10.1016/j.carres.2012.11.022

Mende, D. R., Waller, A. S., Sunagawa, S., Järvelin, A. I., Chan, M. M., Arumugam, M., et al. (2012). Assessment of metagenomic assembly using simulated next generation sequencing data. PLoS One 7:e31386. doi: 10.1371/ journal.pone.0031386

Miller, G. L. (1959). Use of dinitrosalicylic acid reagent for determination of reducing sugar. Anal. Chem. 31, 426-428. doi: 10.1021/ac60147a030

Nielsen, H. B., Almeida, M., Juncker, A. S., Rasmussen, S., Li, J., Sunagawa, S., et al. (2014). Identification and assembly of genomes and genetic elements in complex metagenomic samples without using reference genomes. Nat. Biotechnol. 32, 822-828. doi: 10.1038/nbt.2939

Ohta, Y., Hatada, Y., Miyazaki, M., Nogi, Y., Ito, S., and Horikoshi, K. (2005). Purification and characterization of a novel $\alpha$-agarase from a Thalassomonas sp. Curr. Microbiol. 50, 212-216. doi: 10.1007/s00284-0044435-Z

Ohta, Y., Hatada, Y., Nogi, Y., Li, Z., Ito, S., and Horikoshi, K. (2004). Cloning, expression, and characterization of a glycoside hydrolase family $86 \beta$-agarase from a deep-sea microbulbifer -like isolate. Appl. Microbiol. Biotechnol. 66, 266-275. doi: 10.1007/s00253-004-1757-5

Paës, G., Berrin, J. G., and Beaugrand, J. (2012). GH11 xylanases: structure/function/properties relationships and applications. Biotechnol. Adv. 30, 564-592. doi: 10.1016/j.biotechadv.2011.10.003

Peng, H., Wang, Y., Zheng, Y., Wang, M., Xiao, Y., and Gao, Y. (2013). $\alpha$-Amylase (AmyP) of glycoside hydrolase subfamily GH13_37 is resistant to various toxic compounds. J. Mol. Catal. B 98, 114-118. doi: 10.1016/j.molcatb.2013. 10.003

Petersen, T. N., Brunak, S., von Heijne, G., and Nielsen, H. (2011). SignalP 4.0: discriminating signal peptides from transmembrane regions. Nat. Methods 8 , 785-786. doi: 10.1038/nmeth.1701

Pfennig, N., and Biebl, H. (1976). Desulfuromonas acetoxidans gen. nov. and sp. nov., a new anaerobic, sulfur-reducing, acetate-oxidizing bacterium. Arch. Microbiol. 110, 3-12. doi: 10.1007/BF00416962

Pope, P. B., and Moran, N. A. (2010). Adaptation to herbivory by the Tammar wallaby includes bacterial and glycoside hydrolase profiles different from other herbivores. Proc. Natl. Acad. Sci. U.S.A. 107, 14793-14798. doi: 10.1073/pnas. 1005297107

Qin, J., Li, R., Raes, J., Arumugam, M., Burgdorf, K. S., Manichanh, C., et al. (2010). A human gut microbial gene catalogue established by metagenomic sequencing. Nature 464, 59-65. doi: 10.1038/nature08821

Roden, E. E., and Lovley, D. R. (1993). Dissimilatory Fe(III) reduction by the marine microorganism Desulfuromonas acetoxidans. Appl. Environ. Microbiol. 59, 734-742.

Roesijadi, G., Jones, S. B., Snowden-Swan, L. J., and Zhu, Y. (2010). Macroalgae as a biomass feedstock: a preliminary analysis, PNNL 19944. Richland, WA: Pacific Northwest National Laboratory. doi: 10.2172/1006310 
Rusch, D. B., Halpern, A. L., Sutton, G., Heidelberg, K. B., Williamson, S., Yooseph, S., et al. (2007). The sorcerer II global ocean sampling expedition: northwest atlantic through eastern tropical pacific. PLoS Biol. 5:e77. doi: 10. 1371/journal.pbio.0050077

Sandgren, M., Ståhlberg, J., and Mitchinson, C. (2005). Structural and biochemical studies of GH family 12 cellulases: improved thermal stability, and ligand complexes. Prog. Biophys. Mol. Biol. 89, 246-291. doi: 10.1016/j.pbiomolbio. 2004.11.002

Sheydina, A., Eberhardt, R. Y., Rigden, D. J., Chang, Y., Li, Z., Zmasek, C. C., et al. (2014). Structural genomics analysis of uncharacterized protein families overrepresented in human gut bacteria identifies a novel glycoside hydrolase. BMC Bioinformatics 15:112. doi: 10.1186/1471-2105-15-112

Shome, B. R., Shome, R., Ahlawat, S. P. S., and Verma, N. D. (2000). Agar depolymerizing (agarolytic) bacteria isolated from mangrove soil samples of Andaman. Curr. Sci. 79, 696-697.

Slaby, B. M., Hackl, T., Horn, H., Bayer, K., and Hentschel, U. (2017). Metagenomic binning of a marine sponge microbiome reveals unity in defense but metabolic specialization. ISME J. 11, 2465-2478. doi: 10.1038/ismej.2017.101

Štefan, J., Kuchtová, A., and Petroviěová, S. (2015). A novel GH13 subfamily of $\alpha$-amylases with a pair of tryptophans in the helix $\alpha 3$ of the catalytic TIMbarrel, the LPDlx signature in the conserved sequence region $\mathrm{V}$ and a conserved aromatic motif at the C-terminus. Biologia 70, 1284-1294.

Sunagawa, S., Coelho, L. P., Chaffron, S., Kultima, J. R., Labadie, K., Salazar, G., et al. (2015). Ocean plankton. Structure and function of the global ocean microbiome. Science 348:1261359.

Suzuki, H., Sawai, Y., Suzuki, T., and Kawai, K. (2003). Purification and characterization of an extracellular $\beta$-agarase from Bacillus sp. MK03. J. Biosci. Bioeng. 95, 328-334. doi: 10.1016/S1389-1723(03)80063-4

R Core Team (2016). R: A language and environment for statistical computing. Vienna: R Foundation for Statistical Computing. doi: 10.1186/2191-0855-3-65

Thompson, C. E., Beys-da-Silva, W. O., Santi, L., Berger, M., Vainstein, M. H., and Vasconcelos, A. T. R. (2013). A potential source for cellulolytic enzyme discovery and environmental aspects revealed through metagenomics of Brazilian mangroves. AMB Express 3:65. doi: 10.3390/md8010200

Ting, F. X., and Moo, K. S. (2010). Agarase: review of major sources, categories, purification method, enzyme characteristics and applications. Mar. Drugs 8, 200-218. doi: 10.1016/j.biochi.2013.05.018

Tishkov, V. I., Gusakov, A. V., Cherkashina, A. S., and Sinitsyn, A. P. (2013). Engineering the $\mathrm{pH}$-optimum of activity of the GH12 family endoglucanase by site-directed mutagenesis. Biochimie 95, 1704-1710.

Uchiyama, T., Igarashi, K., and Samejima, M. (2017). "P-53 Processive movement observation of GH6 cellulases", in Proceedings of the Conference on Biomass Science, Tokyo. doi: 10.1094/MPMI-02-12-0043-R

Van, V. B., Itoh, K., Nguyen, Q. B., Tosa, Y., and Nakayashiki, H. (2012). Cellulases belonging to glycoside hydrolase families 6 and 7 contribute to the virulence of Magnaporthe oryzae. Mol. Plant Microbe Interact. 25, 1135-1141. doi: 10.1007/ BF01868635

von Heijne, G. (1990). The signal peptide. J. Membr. Biol. 115, 195-201. doi: 10.1023/B:JAPH.0000047944.40463.e6

Wang, J., Jiang, X., Mou, H., and Guan, H. (2004). Anti-oxidation of agar oligosaccharides produced by agarase from a marine bacterium. J. Appl. Phycol. 16, 333-340. doi: 10.1038/srep44252

Wang, W., Liu, P., Hao, C., Wu, L., Wan, W., and Mao, X. (2017). Neoagaro-oligosaccharide monomers inhibit inflammation in LPS-stimulated macrophages through suppression of MAPK and NF-кB pathways. Sci. Rep. 7:44252. doi: 10.1016/j.biortech.2012.02.092

Wang, Y., Fu, Z., Huang, H., Zhang, H., Yao, B., Xiong, H., et al. (2012). Improved thermal performance of Thermomyces lanuginosus GH11 xylanase by engineering of an N-terminal disulfide bridge. Bioresour. Technol. 112, 275-279. doi: 10.1126/science.1214547

Wargacki, A. J., Leonard, E., Win, M. N., Regitsky, D. D., Santos, C. N. S., Kim, P. B., et al. (2012). An engineered microbial platform for direct biofuel production from brown macroalgae. Science 335, 308-313. doi: 10.1038/nature06269

Warnecke, F., Luginbühl, P., Ivanova, N., Ghassemian, M., Richardson, T. H., Stege, J. T., et al. (2007). Metagenomic and functional analysis of hindgut microbiota of a wood-feeding higher termite. Nature 450, 560-565. doi: 10 . 1016/j.molcatb.2017.02.003

Watanabe, T., Kashimura, K., and Kirimura, K. (2017). Purification, characterization and gene identification of a $\alpha$-Neoagarooligosaccharide hydrolase from an alkaliphilic bacterium Cellvibrio sp. WU-0601. J. Mol. Catal. B 133, S328-S336. doi: 10.1371/journal.pone.0164082

Wu, P., Xiong, X., Xu, Z., Lu, C., Cheng, H., Lyu, X., et al. (2016). Bacterial communities in the rhizospheres of three mangrove tree species from Beilun Estuary, China. PLoS One 11:e0164082. doi: 10.1371/journal.pone.01 64082

Wu, Y. -R., Zhang, M., Zhong, M., and Hu, Z. (2017). Synergistic enzymatic saccharification and fermentation of agar for biohydrogen production. Bioresour. Technol. 241, 369-373. doi: 10.3389/fmicb.2015. 01410

Xiao, Y., Zheng, Y., Wu, S., Zhang, E. H., Chen, Z., Liang, P., et al. (2015). Pyrosequencing reveals a core community of anodic bacterial biofilms in bioelectrochemical systems from China. Front. Microbiol. 6:1410. doi: 10.3389/ fmicb.2015.01410

Yanagisawa, M., Kawai, S., and Murata, K. (2013). Strategies for the production of high concentrations of bioethanol from seaweeds: production of high concentrations of bioethanol from seaweeds. Bioengineered 4, 224-235. doi: 10.1186/s13068-016-0557-3

Yang, C., Xia, Y., Qu, H., Li, A. D., Liu, R., Wang, Y., et al. (2016). Discovery of new cellulases from the metagenome by a metagenomics-guided strategy. Biotechnol. Biofuels 9:138. doi: 10.1111/1462-2920.12607

Yun, E. J., Lee, S., Kim, H. T., Pelton, J. G., Kim, S., Ko, H. J., et al. (2015a). The novel catabolic pathway of 3, 6-anhydro-L-galactose, the main component of red macroalgae, in a marine bacterium. Environ. Microbiol. 17, 1677-1688. doi: 10.1111/1462-2920.12607

Yun, E. J., Saeyoung, L., Heetaek, K., Pelton, J. G., Sooah, K., Hyeokjin, K., et al. (2015b). The novel catabolic pathway of 3,6-anhydro-L-galactose, the main component of red macroalgae, in a marine bacterium. Environ. Microbiol. 17, 1677-1688. doi: 10.1016/S0076-6879(05)97007-9

Zengler, K., Walcher, M., Clark, G., Haller, I., Toledo, G., Holland, T., et al. (2005). High-throughput cultivation of microorganisms using microcapsules. Methods Enzymol. 397, 124-130. doi: 10.1016/j.biortech.2011.06.085

Zhang, J., Tuomainen, P., Siika-Aho, M., and Viikari, L. (2011). Comparison of the synergistic action of two thermostable xylanases from GH families 10 and 11 with thermostable cellulases in lignocellulose hydrolysis. Bioresour. Technol. 102, 9090-9095. doi: 10.1104/pp.15.01690

Zhang, R., Tucker, M. R., Burton, R. A., Shirley, N. J., Little, A., Morris, J., et al. (2016). The dynamics of transcript abundance during cellularisation of developing barley endosperm. Plant Physiol. 170, 1549-1565. doi: 10.1104/pp. 15.01690

Zhang, X., Liu, W., Zhang, G., Jiang, L., and Han, X. (2015). Mechanisms of soil acidification reducing bacterial diversity. Soil Biol. Biochem. 81, 275-281. doi: 10.1016/j.soilbio.2014.11.004

Zhang, Y.H.P., and Lynd, L.R. (2003). Cellodextrin preparation by mixed-acid hydrolysis and chromatographic separation. Anal. Biochem. 322, 225-232. doi: 10.1016/j.ab.2003.07.021

Zhao, L., Zhou, T., Li, X., Fan, S., and You, L. (2013). Expression and characterization of GH3 $\beta$-Glucosidase from Aspergillus niger NL-1 with high specific activity, glucose inhibition and solvent tolerance. Microbiology 82, 356-363. doi: 10.1134/S0026261713030181

Zheng, J., Xiao, X., Zhang, Q., Mao, L., Yu, M., and Xu, J. (2015). The placental microbiome varies in association with low birth weight in full-term neonates. Nutrients 7, 6924-6937. doi: 10.3390/nu7085315

Zhu, Y., Zhao, R., Xiao, A., Li, L., Jiang, Z., Chen, F., et al. (2016). Characterization of an alkaline $\beta$-agarase from Stenotrophomonas sp. NTa and the enzymatic hydrolysates. Int. J. Biol. Macromol. 86, 525-534. doi: 10.1016/j.ijbiomac.2016. 01.106

Conflict of Interest Statement: The authors declare that the research was conducted in the absence of any commercial or financial relationships that could be construed as a potential conflict of interest.

Copyright $\odot 2018$ Qu, Lin, Zhang, Di, Gao and Zeng. This is an open-access article distributed under the terms of the Creative Commons Attribution License (CC BY). The use, distribution or reproduction in other forums is permitted, provided the original author(s) and the copyright owner(s) are credited and that the original publication in this journal is cited, in accordance with accepted academic practice. No use, distribution or reproduction is permitted which does not comply with these terms. 